\title{
Radon Inversion Problem for Holomorphic Functions on Circular, Strictly Convex Domains
}

\author{
P. Pierzchała ${ }^{1}$ (D) P. $\operatorname{Kot}^{1}$
}

Received: 30 July 2020 / Accepted: 20 May 2021 / Published online: 2 June 2021

(c) The Author(s) 2021

\begin{abstract}
In this paper we study the so-called Radon inversion problem in bounded, circular, strictly convex domains with $\mathcal{C}^{2}$ boundary. We show that given $p>0$ and a strictly positive, continuous function $\Phi$ on $\partial \Omega$, by use of homogeneous polynomials it is possible to construct a holomorphic function $f \in \mathcal{O}(\Omega)$ such that $\int_{0}^{1}|f(z t)|^{p} d t=\Phi(z)$ for all $z \in \partial \Omega$. In our approach we make use of so-called lacunary $K$-summing polynomials (see definition below) that allow us to construct solutions with in some sense extremal properties.
\end{abstract}

Keywords Radon inversion problem - Divergent Taylor series · Boundary behaviour of holomorphic functions of several complex variables · Inner functions

Mathematics Subject Classification Primary 32A40 - Secondary 32A0

\section{Introduction}

In general, Radon inversion problem is to reconstruct a function on the basis of the values of its integrals over some subset of submanifolds of its domain. Here we consider bounded, circular, strictly convex domains and we are interested in finding a holomorphic function such that its radial integrals are equal to the values of some given strictly positive, continuous function on the boundary. More precisely, let $\Omega \subset \mathbb{C}^{v}$ be a bounded, circular, strictly convex domain with $\mathcal{C}^{2}$ boundary. Fix $p>0$. For a

\section{Communicated by David Kimsey.}

P. Pierzchała

paulina.pierzchala@agh.edu.pl

P. Kot

piotr.kot@agh.edu.pl

1 Faculty of Applied Mathematics, AGH University of Science and Technology, al. Mickiewicza 30, 30-059 Kraków, Poland 
holomorphic function $f \in \mathcal{O}(\Omega)$ we may consider the integral operator $\mathcal{R}^{p}$ defined as follows

$$
\mathcal{R}^{p}(f)(z):=\int_{0}^{1}|f(z t)|^{p} d t, \quad z \in \bar{\Omega}
$$

and called Radon operator. Then for a given strictly positive, continuous function $\Phi: \partial \Omega \longmapsto \mathbb{R}_{+}$we look for a function $f \in \mathcal{O}(\Omega)$ such that

$$
\mathcal{R}^{p}(f)=\Phi \text { on } \partial \Omega
$$

Slightly different formulation of the Radon inversion problem in terms of considered integrals for the operator $\mathcal{R}^{p}$ was described in [7] and solved at almost all boundary points with respect to a given probability measure on the boundary of the domain. Moreover, in the same paper it was observed that Radon inversion problem for holomorphic functions is similar to construction of inner functions in several variables. The radial limits that exist almost everywhere on the boundary are replaced by the Radon operator. However, the Radon operator $\mathcal{R}^{p}$ is well-defined at all boundary points, so the aim of the present paper is to solve (1) for all $z \in \partial \Omega$. For the first two independent constructions of a non-constant inner function in several variables see [1] and [9].

To achieve our aim we make use of homogeneous polynomials constructed in [6]. Historically they are a generalization of Ryll-Wojtaszczyk polynomials (see [11]). For our purpose we add some properties to the polynomials from [6] to obtain, as we call them, lacunary $K$-summing polynomials (see definition below). They will form generators that enable us to construct by induction a solution to (1).

Then we intend to study properties of the obtained solution. In our approach, the solution is clearly unbounded on the boundary of the domain. Nevertheless, more interesting properties might be seen by considering a linear operator $\mathcal{S}^{p}$ for holomorphic functions, $p>0$. If $\left\{u_{k}\right\}_{k \in \mathbb{N}}$ is a sequence of homogeneous polynomials of degree $n_{k} \in \mathbb{N}$ respectively, then the operator $\mathcal{S}^{p}$ is defined as follows

$$
\mathcal{S}^{p}: \mathcal{O}(\Omega) \ni \sum_{k=1}^{\infty} u_{k} \longmapsto \sum_{k=1}^{\infty} \frac{u_{k}}{\sqrt[p]{p n_{k}+1}} \in \mathcal{O}(\Omega)
$$

First of all, we construct a solution $f$ to (1) such that $\mathcal{S}^{p}(f)$ is continuous up to the boundary for any $p>0$. Then we show that one may find another solution $g$ such that all slice functions of $\mathcal{S}^{p}(g)$ have divergent series of Taylor coefficients with every exponent $s<\min \{1, p\}$. Moreover, if $p \leq 1$, then $\mathcal{S}^{p}(g) \in \mathcal{C}(\bar{\Omega})$ and for $p \in(1,2]$, function $\mathcal{S}^{p}(g)$ is square-integrable on all circles $z \partial \mathbb{D}, \quad z \in \partial \Omega$. This is a similar result to the ones obtained for functions in the ball algebra in [12] and for inner functions in [2] and [3]. In some sense these two approaches give us flexibility and diversity in solving Radon inversion problem and might be useful in view of constructing holomorphic functions with prescribed boundary behaviour. 


\section{Notation and Preliminaries}

In this paper we set the following notation. Let $\sigma, \mu$ and $\lambda$ be normalized Lebesgue measures on $\partial \Omega, \Omega$ and $\partial \mathbb{D}$, respectively, i.e. $\sigma(\partial \Omega)=1, \mu(\Omega)=1$ and $\lambda(\partial \mathbb{D})=1$. Let $\mathcal{C}_{M}(\bar{\Omega})$ stand for the space of continuous functions in $\bar{\Omega}$ and bounded by $M$. Let us denote $L_{1}$ norm on circles $z \partial \mathbb{D}, z \in \partial \Omega$, by $\|f\|_{1, z \partial \mathbb{D}}:=\int_{\partial \mathbb{D}}|f(z \omega)| d \lambda(\omega)$ and supremum norm $\|f\|_{T}:=\sup _{z \in T}|f(z)|$. For $z \in \partial \Omega$ denote by $f_{z}: \mathbb{D} \ni \omega \longmapsto$ $f(\omega z)$ the slice function for $f$. Recall that a polynomial $P$ is said to be of order $k$ if $\frac{\partial^{|\alpha|}}{\partial z^{\alpha}} P(0)=0$ for any multi-index $\alpha$ such that $|\alpha|<k$ and we write $\operatorname{ord}(P)=k$. Finally, for $p>0$ and $q=\max \{p, 1\}$ we define the space

$$
\mathcal{H} \mathcal{R}^{p}(\Omega):=\left\{f \in \mathcal{O}(\Omega): \sup _{z \in \partial \Omega} \mathcal{R}^{p}(f)(z)<\infty\right\}
$$

equipped with the metric $d(f, g):=\sup _{z \in \partial \Omega}\left(\mathcal{R}^{p}(f-g)(z)\right)^{\frac{1}{q}}$, where $f, g \in \mathcal{H} \mathcal{R}^{p}(\Omega)$. This space is complete as we shall show it in the following proposition.

Proposition 2.1 The space $\left(\mathcal{H}^{p}(\Omega), d\right)$ is complete for any $p>0$. Moreover, if $f_{n} \underset{n \rightarrow \infty}{\stackrel{d}{\longrightarrow}} f$ in $\mathcal{H R}^{p}(\Omega)$, then $\mathcal{R}^{p}\left(f_{n}\right) \rightrightarrows \mathcal{R}^{p}(f)$ on $\partial \Omega$ as $n \rightarrow \infty$.

Proof Let $\left\{f_{n}\right\}_{n \in \mathbb{N}}$ be a Cauchy sequence in $\mathcal{H} \mathcal{R}^{p}(\Omega)$. Set $\varepsilon>0$. There exists $N \in \mathbb{N}$ such that

$$
\left(\mathcal{R}^{p}\left(f_{m}-f_{n}\right)(z)\right)^{\frac{1}{q}}<\varepsilon \quad \forall_{m, n \geq N} \quad \forall_{z \in \partial \Omega}
$$

which implies that

$$
\int_{0}^{1}\left|\left(f_{m}-f_{n}\right)(z t)\right|^{p} d t<\varepsilon^{q} \quad \forall_{m, n \geq N} \quad \forall_{z \in \partial \Omega}
$$

Now we may integrate (2) over $\partial \Omega$ to obtain that

$$
\int_{\partial \Omega} \int_{0}^{1}\left|\left(f_{m}-f_{n}\right)(z t)\right|^{p} d t d \sigma(z)<\varepsilon^{q} \quad \forall_{m, n \geq N} .
$$

Since $f_{m}-f_{n}$ is a holomorphic function, on the basis of [10] (Prop. 1.5.4.), function $\left|f_{m}-f_{n}\right|^{p}$ is subharmonic. Let $K$ be a compact subset of $\Omega, w \in K$ and $r=$ $\frac{1}{2} \operatorname{dist}(K, \partial \Omega)$, so $\overline{\mathbb{B}(w, r)} \subset \Omega$. The sub-mean value theorem applied to the function 
$\left|f_{m}-f_{n}\right|^{p}$ and $\mathbb{B}(w, r)$ gives the following estimate

$$
\begin{aligned}
\left|\left(f_{m}-f_{n}\right)(w)\right|^{p} & \leq \frac{1}{\mu(\overline{\mathbb{B}(w, r)})} \int_{\mathbb{B}(w, r)}\left|\left(f_{m}-f_{n}\right)(\zeta)\right|^{p} d \mu(\zeta) \\
& \leq \frac{1}{r^{2 v}} \int_{\partial \Omega} \int_{0}^{1}\left|\left(f_{m}-f_{n}\right)(z t)\right|^{p} d t d \sigma(z)<\frac{\varepsilon^{q}}{r^{2 v}}
\end{aligned}
$$

for any $m, n \geq N$. In particular,

$$
\sup _{w \in K}\left|\left(f_{m}-f_{n}\right)(w)\right|^{p}<\frac{\varepsilon^{q}}{r^{2 v}} \quad \forall_{m, n \geq N} .
$$

This implies that $\left\{f_{n}\right\}_{n \in \mathbb{N}}$ converges locally uniformly on compact subsets of $\Omega$. Let $f$ be the limit function for the sequence $\left\{f_{n}\right\}_{n \in \mathbb{N}}$. Since $f_{n}$ are holomorphic, so is $f$.

Now we shall show that $f_{n} \stackrel{d}{\longrightarrow} f$ and $f \in \mathcal{H} \mathcal{R}^{p}(\Omega)$. It follows from (2) that

$$
\int_{0}^{\delta}\left|\left(f_{m}-f_{n}\right)(z t)\right|^{p} d t<\varepsilon^{q} \quad \forall_{\delta \in(0,1)} \quad \forall_{m, n \geq N} \quad \forall_{z \in \partial \Omega} .
$$

Since $f_{m} \longrightarrow f$ locally uniformly we may pass with $m$ to the limit in (3) to obtain that

$$
\int_{0}^{\delta}\left|\left(f-f_{n}\right)(z t)\right|^{p} d t \leq \varepsilon^{q} \quad \forall_{\delta \in(0,1)} \quad \forall_{n \geq N} \quad \forall_{z \in \partial \Omega}
$$

Above inequality holds for any $\delta \in(0,1)$, so we get that

$$
\int_{0}^{1}\left|\left(f-f_{n}\right)(z t)\right|^{p} d t \leq \varepsilon^{q} \quad \forall_{n \geq N} \quad \forall_{z \in \partial \Omega} .
$$

Moreover, by triangle inequality,

$$
\sup _{z \in \partial \Omega}\left(\mathcal{R}^{p}(f)(z)\right)^{\frac{1}{q}} \leq \sup _{z \in \partial \Omega}\left(\mathcal{R}^{p}\left(f-f_{n}\right)(z)\right)^{\frac{1}{q}}+\sup _{z \in \partial \Omega}\left(\mathcal{R}^{p}\left(f_{n}\right)(z)\right)^{\frac{1}{q}}<\infty \quad \forall_{n \geq N} .
$$

Therefore $f_{n} \underset{n \rightarrow \infty}{\stackrel{d}{\longrightarrow}} f$ and $f \in \mathcal{H} \mathcal{R}^{p}(\Omega)$.

If $f_{n} \underset{n \rightarrow \infty}{\stackrel{d}{\longrightarrow}} f$, then $d\left(f, f_{n}\right) \underset{n \rightarrow \infty}{\longrightarrow} 0$. Triangle inequality implies that

$$
\sup _{z \in \partial \Omega}\left(\left(\mathcal{R}^{p}(f)(z)\right)^{\frac{1}{q}}-\left(\mathcal{R}^{p}\left(f_{n}\right)(z)\right)^{\frac{1}{q}}\right) \leq \sup _{z \in \partial \Omega}\left(\mathcal{R}^{p}\left(f-f_{n}\right)(z)\right)^{\frac{1}{q}} \underset{n \rightarrow \infty}{\longrightarrow} 0 .
$$

Hence $\left(\mathcal{R}^{p}\left(f_{n}\right)\right)^{\frac{1}{q}} \rightrightarrows\left(\mathcal{R}^{p}(f)\right)^{\frac{1}{q}}$ on $\partial \Omega$ as $n \rightarrow \infty$ and consequently that $\mathcal{R}^{p}\left(f_{n}\right) \rightrightarrows$ $\mathcal{R}^{p}(f)$ on $\partial \Omega$ as $n \rightarrow \infty$. 
In the construction of a solution to the Radon inversion problem we will use the following theorem from [4]. It provides a collection of holomorphic and continuous up to the boundary functions that in some sense together approximate a given strictly positive, continuous function on $\partial \Omega$. It is important to emphasise that the number of elements in this set of functions does not depend on the function we want to approximate.

Theorem 2.2 ([4], Theorem 3.2) There exists a natural number $N=N(\partial \Omega)$ such that if $\varepsilon \in(0,1), T$ is a compact subset of $\Omega, h$ is a continuous strictly positive function on $\partial \Omega$, then there exist holomorphic functions $f_{1}, \ldots, f_{N} \in \mathcal{O}(\Omega) \cap \mathcal{C}(\bar{\Omega})$ such that

1. $\left\|f_{m}\right\|_{T} \leq \varepsilon, \quad m=1, \ldots, N$

2. $\frac{h}{2}<\max _{1 \leq m \leq N}\left|f_{m}\right|<h$ on $\partial \Omega$.

As it is observed in [8], functions in Theorem 2.2 may be replaced with polynomials.

\section{Lacunary K-Summing Polynomials}

As we said before, the main tool in our approach to solve the Radon inversion problem is lacunary $K$-summing polynomials. Hereafter, we give a precise definition and then we study useful properties of lacunary $K$-summing polynomials, in particular how they behave composed with operators $\mathcal{R}^{p}$ and $\mathcal{S}^{p}$ and what relation there is between these operators.

Definition 3.1 For a given $K \in \mathbb{N}$, a polynomial $Q$ is called a $K$-summing polynomial, if it has a homogeneous expansion $\sum_{j=1}^{K} u_{j}$, where $\operatorname{deg}\left(u_{j}\right)=n_{j} \in \mathbb{N}, \quad j=$ $1,2, \ldots, K$, and satisfies the following conditions
i) $\max _{1 \leq j \leq K}\left|u_{j}(z)\right| \leq 1$ for $z \in \partial \Omega$
ii) $\frac{1}{2} \operatorname{deg}(Q) \leq \operatorname{ord}(Q)=n_{1}<n_{2}<\cdots<n_{K}=\operatorname{deg}(Q)$.

Moreover, $Q$ is said to be a lacunary $K$-summing polynomial, if it additionally possesses properties $i i i)$ and $i v$ )

iii) $\max _{1 \leq j \leq K}\left|u_{j}(z)\right| \geq \frac{1}{2}$ for $z \in \partial \Omega$

iv) $\sqrt[K]{2}<\frac{n_{j+1}}{n_{j}}<2, \quad j=1,2, \ldots, K-1$.

To prove the existence of lacunary $K$-summing polynomials we need the following theorem from [6].

Theorem 3.2 ([6], Theorem 2.5) There exists $K=K(\partial \Omega) \in \mathbb{N}$ such that there exists $N_{0} \in \mathbb{N}$ such that for all integers $N \geq N_{0}$ and $n_{1}, \ldots, n_{K} \in \mathbb{N}$ with $N \leq n_{1} \leq \cdots \leq$ $n_{K} \leq 2 N$ there exist homogeneous polynomials $u_{1}, \ldots, u_{K}$ of degrees $n_{1}, \ldots, n_{K}$, respectively, such that $\frac{1}{2}<\max _{1 \leq j \leq K}\left|u_{j}(z)\right|<1$ for all $z \in \partial \Omega$. 
Corollary 3.3 There exists $K=K(\partial \Omega) \in \mathbb{N}$ for which there exists $N_{K} \in \mathbb{N}$ such that for any $N \geq N_{K}$ there exists a lacunary $K$-summing polynomial of degree $N$.

Proof Take $K=K(\partial \Omega) \in \mathbb{N}$ and $N_{0} \in \mathbb{N}$ from Theorem 3.2. Choose such a big $N_{K} \geq 2 N_{0}$ that for $N \geq N_{K}$ there exists a $K$-tuple of integers $\frac{1}{2} N<n_{1}<n_{2}<$ $\cdots<n_{K}=N$ that satisfy $\sqrt[K]{2}<\frac{n_{j+1}}{n_{j}}<2$ for $j=1,2, \ldots, K-1$. For such $N$ and $n_{1}, n_{2}, \ldots, n_{K}$ by Theorem 3.2 , there exist homogeneous polynomials $u_{1}, u_{2}, \ldots, u_{K}$ of degrees $n_{1}, n_{2}, \ldots, n_{K}$, respectively, such that $\frac{1}{2}<\max _{1 \leq j \leq K}\left|u_{j}\right|<1$ on $\partial \Omega$. Then observe that $\sum_{j=1}^{K} u_{j}$ is a lacunary $K$-summing polynomial of degree $N$.

Lemma 3.4 Let $p>0, \varepsilon>0, M>0$ and $K \in \mathbb{N}$. There exists $N_{0} \in \mathbb{N}$ such that for any $K$-summing polynomial $Q$ of degree $N \geq N_{0}$ and $f, g \in \mathcal{C}_{M}(\bar{\Omega})$ the following inequalities

$$
\mathcal{R}^{p}(f Q)+\mathcal{R}^{p}(g)-\varepsilon<\mathcal{R}^{p}(f Q+g)<\mathcal{R}^{p}(f Q)+\mathcal{R}^{p}(g)+\varepsilon
$$

hold in $\bar{\Omega}$.

Proof Let $q=\max \{p, 1\}$ and $k \in \mathbb{N}$ be such that $k \geq p$. We may choose $N_{0} \in \mathbb{N}$ such that for any $N \geq N_{0}$ we have $\frac{M^{p} \overline{K^{q}}}{\frac{1}{2} p N+1}<\frac{\varepsilon}{8}$ and $\sum_{m=1}^{k-1}\left(\begin{array}{l}k \\ m\end{array}\right)^{\frac{p}{k}} M^{p} K^{m} \frac{k}{\frac{1}{2} N p+k}<\frac{\varepsilon}{2}$. We will show that such $N_{0}$ fulfills the requirements to be chosen for Lemma 3.4.

Since $Q$ is a $K$-summing polynomial of degree $N \geq N_{0}$, let $Q=\sum_{j=1}^{K} u_{j}$ be its homogeneous expansion of degrees $n_{1}, \ldots, n_{K}$, respectively, such that $\frac{1}{2} N \leq n_{1}<$ $\cdots<n_{K}=N$. For simplicity denote by $F:=f Q$. Let $\delta \in(0,1)$ be such that $\int_{\delta}^{1}|g(z t)|^{p} d t \leq \frac{\varepsilon}{8}$. Then observe that for $z \in \bar{\Omega}$ we have

$$
\begin{aligned}
\left(\int_{0}^{\delta}|F(z t)|^{p} d t\right)^{\frac{1}{q}} & =\left(\int_{0}^{\delta}\left|f(z t) \sum_{j=1}^{K} t^{n_{j}} u_{j}(z)\right|^{p} d t\right)^{\frac{1}{q}} \\
& \leq M^{\frac{p}{q}} \sum_{j=1}^{K}\left(\left|u_{j}(z)\right|^{p} \int_{0}^{\delta} t^{p n_{j}} d t\right)^{\frac{1}{q}} \\
& \leq M^{\frac{p}{q}} \sum_{j=1}^{K}\left(\frac{\delta n_{j}+1}{p n_{j}+1}\right)^{\frac{1}{q}} \leq\left(M^{p} K^{q} \frac{\delta^{\frac{1}{2} p N+1}}{\frac{1}{2} p N+1}\right)^{\frac{1}{q}}<\left(\frac{\varepsilon}{8}\right)^{\frac{1}{q}} .
\end{aligned}
$$


Hence $\int_{0}^{\delta}|F(z t)|^{p} d t<\frac{\varepsilon}{8}$. Since $\frac{p}{k} \leq 1$, we are able to make use of triangle inequality to obtain the following estimates

$$
\begin{aligned}
\mathcal{R}^{\frac{p}{k}}\left(F^{k}+g^{k}\right)(z) & =\int_{0}^{1}\left|\left(F^{k}+g^{k}\right)(z t)\right|^{\frac{p}{k}} d t \leq \int_{0}^{1}|F(z t)|^{p} d t+\int_{0}^{1}|g(z t)|^{p} d t \\
& =\mathcal{R}^{p}(F)(z)+\mathcal{R}^{p}(g)(z)
\end{aligned}
$$

and

$$
\begin{aligned}
\mathcal{R}^{\frac{p}{k}}\left(F^{k}+g^{k}\right)(z)= & \int_{0}^{1}\left|\left(F^{k}+g^{k}\right)(z t)\right|^{\frac{p}{k}} d t=\int_{0}^{\delta}\left|\left(F^{k}+g^{k}\right)(z t)\right|^{\frac{p}{k}} d t \\
& +\int_{\delta}^{1}\left|\left(F^{k}+g^{k}\right)(z t)\right|^{\frac{p}{k}} d t \\
\geq & \int_{\delta}^{1}|F(z t)|^{p} d t-\int_{0}^{\delta}|F(z t)|^{p} d t \\
& +\int_{0}^{\delta}|g(z t)|^{p} d t-\int_{\delta}^{1}|g(z t)|^{p} d t \\
= & \mathcal{R}^{p}(F)(z)+\mathcal{R}^{p}(g)(z)-2 \int_{0}^{\delta}|F(z t)|^{p} d t-2 \int_{\delta}^{1}|g(z t)|^{p} d t \\
> & \mathcal{R}^{p}(F)(z)+\mathcal{R}^{p}(g)(z)-\frac{\varepsilon}{2} .
\end{aligned}
$$

If $0<p \leq 1$, then $k=1$ does the job. For $p>1$ we need a little bit more effort to make. For shortness, denote by

$$
\mathcal{I}_{k}^{p}(F, g)(z):=\int_{0}^{1}\left|\sum_{m=1}^{k-1}\left(\begin{array}{l}
k \\
m
\end{array}\right)(F(z t))^{m}(g(z t))^{k-m}\right|^{\frac{p}{k}} d t .
$$

Then observe that for $z \in \bar{\Omega}$

$$
\begin{aligned}
\mathcal{I}_{k}^{p}(F, g)(z) & \leq \sum_{m=1}^{k-1}\left(\begin{array}{c}
k \\
m
\end{array}\right)^{\frac{p}{k}} \int_{0}^{1}\left|f(z t) \sum_{j=1}^{K} u_{j}(z t)\right|^{\frac{m p}{k}}|g(z t)|^{\frac{(k-m) p}{k}} d t \\
& \leq \sum_{m=1}^{k-1}\left(\begin{array}{c}
k \\
m
\end{array}\right)^{\frac{p}{k}} M^{\frac{m p}{k}} M^{\frac{p(k-m)}{k}}\left(\sum_{j=1}^{K}\left|u_{j}(z)\right|^{\frac{p}{k}} \int_{0}^{1} t^{\frac{p n_{j}}{k}} d t\right)^{m} \\
& \leq \sum_{m=1}^{k-1}\left(\begin{array}{c}
k \\
m
\end{array}\right)^{\frac{p}{k}} M^{p}\left(\sum_{j=1}^{\frac{K}{p n_{j}+k}}\right)^{m} \leq \sum_{m=1}^{k-1}\left(\begin{array}{c}
k \\
m
\end{array}\right)^{\frac{p}{k}} M^{p}\left(K \frac{k}{\frac{1}{2} p N+k}\right)^{m} \\
& \leq \sum_{m=1}^{k-1}\left(\begin{array}{c}
k \\
m
\end{array}\right)^{\frac{p}{k}} M^{p} K^{m} \frac{k}{\frac{1}{2} N p+k}<\frac{\varepsilon}{2} .
\end{aligned}
$$


This implies that for $z \in \bar{\Omega}$

$$
\begin{aligned}
\mathcal{R}^{p}(F+g)(z) & =\int_{0}^{1}\left(|(F+g)(z t)|^{k}\right)^{\frac{p}{k}} d t=\int_{0}^{1}\left|\sum_{m=0}^{k}\left(\begin{array}{c}
k \\
m
\end{array}\right)(F(z t))^{m}(g(z t))^{k-m}\right|^{\frac{p}{k}} d t \\
& \leq \int_{0}^{1}|F(z t)|^{p} d t+\int_{0}^{1}|g(z t)|^{p} d t+\mathcal{I}_{k}^{p}(F, g)(z) \\
& <\mathcal{R}^{p}(F)(z)+\mathcal{R}^{p}(g)(z)+\frac{\varepsilon}{2}
\end{aligned}
$$

and

$$
\begin{aligned}
\mathcal{R}^{p}(F+g)(z) & =\int_{0}^{1}|(F+g)(z t)|^{p} d t=\int_{0}^{1}\left|\sum_{m=0}^{k}\left(\begin{array}{c}
k \\
m
\end{array}\right)(F(z t))^{m}(g(z t))^{k-m}\right|^{\frac{p}{k}} d t \\
{[5 m m] } & \geq \int_{0}^{1}\left|\left(F^{k}+g^{k}\right)(z t)\right|^{\frac{p}{k}} d t-\mathcal{I}_{k}^{p}(F, g)(z) \\
& >\int_{0}^{1}|F(z t)|^{p} d t+\int_{0}^{1}|g(z t)|^{p} d t-\varepsilon \\
& =\mathcal{R}^{p}(F)(z)+\mathcal{R}^{p}(g)(z)-\varepsilon .
\end{aligned}
$$

Above estimates complete the proof.

Lemma 3.5 Let $p>0, \varepsilon>0, M>0$ and $K \in \mathbb{N}$. There exists $N_{0} \in \mathbb{N}$ such that for any $K$-summing polynomial $Q$ of degree $N \geq N_{0}$ and $f \in \mathcal{C}_{M}(\bar{\Omega})$ the following inequalities

$$
|f|^{p} \mathcal{R}^{p}(Q)-\varepsilon \leq \mathcal{R}^{p}(f Q) \leq|f|^{p} \mathcal{R}^{p}(Q)+\varepsilon
$$

are satisfied in $\bar{\Omega}$.

Proof There exists $N_{0} \in \mathbb{N}$ such that for any $N \geq N_{0}$ the following inequalities hold true $\frac{M^{p} K^{p+1}}{\frac{1}{2} N p+1} \leq \frac{\varepsilon}{2}$ and $\frac{1}{2} N p+1>K^{p+1}$. We will show that such $N_{0}$ fulfills the requirements to be chosen for Lemma 3.5.

Let $\sum_{j=1}^{K} u_{j}$ be the homogeneous expansion of the polynomial $Q$, where $u_{1}, \ldots, u_{K}$ are homogeneous polynomials of degrees $n_{1}, \ldots, n_{K}$, respectively, such that $\frac{1}{2} N \leq n_{1}<\cdots<n_{K}=N$. Since $f$ is continuous in $\bar{\Omega}$, there exists $\delta \in(0,1)$ such that for $t \in[\delta, 1]$, if $z \in \bar{\Omega}$, then

$$
|f(z)|^{p}-\frac{\varepsilon}{2} \leq|f(z t)|^{p} \leq|f(z)|^{p}+\frac{\varepsilon}{2}
$$


Let $q=\max \{p, 1\}$. Then observe that by triangle inequality,

$$
\begin{aligned}
\left(\int_{\delta}^{1}|Q(z t)|^{p} d t\right)^{\frac{1}{q}} & =\left(\int_{\delta}^{1}\left|\sum_{j=1}^{K} u_{j}(z t)\right|^{p} d t\right)^{\frac{1}{q}} \\
& \leq \sum_{j=1}^{K}\left(\int_{\delta}^{1} t^{p n_{j}} d t\right)^{\frac{1}{q}} \leq K\left(\frac{1}{\frac{1}{2} N p+1}\right)^{\frac{1}{q}}<1
\end{aligned}
$$

and

$$
\begin{aligned}
\left(|f(z)|^{p} \int_{0}^{\delta}|Q(z t)|^{p} d t\right)^{\frac{1}{q}} & \leq M^{\frac{p}{q}} \sum_{j=1}^{K}\left(\int_{0}^{\delta}\left|u_{j}(z)\right|^{p} t^{p n_{j}} d t\right)^{\frac{1}{q}} \\
& \leq M^{\frac{p}{q}} \sum_{j=1}^{K}\left(\frac{\delta^{p n_{j}+1}}{p n_{j}+1}\right)^{\frac{1}{q}} \\
& \leq\left(M^{p} K^{q} \frac{\delta^{p N+1}}{\frac{1}{2} p N+1}\right)^{\frac{1}{q}} \leq\left(\frac{\varepsilon}{2}\right)^{\frac{1}{q}}
\end{aligned}
$$

Above inequalities yield the conclusion

$$
\begin{aligned}
\mathcal{R}^{p}(f Q)(z) & =\int_{0}^{1}|f(z t) Q(z t)|^{p} d t \geq \int_{\delta}^{1}|f(z t) Q(z t)|^{p} d t \\
& \stackrel{(4)}{\geq}\left(|f(z)|^{p}-\frac{\varepsilon}{2}\right) \int_{\delta}^{1}|Q(z t)|^{p} d t \\
& \stackrel{(5)}{\geq}|f(z)|^{p} \int_{0}^{1}|Q(z t)|^{p} d t-|f(z)|^{p} \int_{0}^{\delta}|Q(z t)|^{p} d t-\frac{\varepsilon}{2} \\
& \stackrel{(6)}{\geq}|f(z)|^{p} \mathcal{R}^{p}(Q)(z)-\varepsilon
\end{aligned}
$$

and

$$
\begin{aligned}
\mathcal{R}^{p}(f Q)(z)= & \int_{0}^{1}|f(z t) Q(z t)|^{p} d t=\int_{\delta}^{1}|f(z t) Q(z t)|^{p} d t+\int_{0}^{\delta}|f(z t) Q(z t)|^{p} d t \\
\stackrel{(4),(6)}{\leq}\left(|f(z)|^{p}+\frac{\varepsilon}{2}\right) \int_{\delta}^{1}|Q(z t)|^{p} d t & \\
& +\frac{\varepsilon}{2} \stackrel{(5)}{\leq}|f(z)|^{p} \int_{0}^{1}|Q(z t)|^{p} d t+\varepsilon \\
= & |f(z)|^{p} \mathcal{R}^{p}(Q)(z)+\varepsilon
\end{aligned}
$$

for $z \in \bar{\Omega}$. This finishes the proof. 
Lemma 3.6 Let $p>0, m \in \mathbb{N}, \eta \in(1,2), \delta \in(0,1)$. There exist $c_{m} \in(0, \delta)$ and $l_{m} \in \mathbb{N}$ such that for any polynomial of the form $P_{m}(t)=\sum_{j=1}^{m} a_{j} t^{n_{j}}$, where $\delta \leq \max _{1 \leq j \leq m}\left|a_{j}\right| \leq 1, n_{1} \geq l_{m}$ and $\eta<\frac{n_{j+1}}{n_{j}}<2$ for $j=1, \ldots, m-1$, the following inequality holds true

$$
\int_{0}^{1} p n_{m}\left|P_{m}(t)\right|^{p} d t \geq c_{m} .
$$

Proof Let $q=\max \{p, 1\}$. We proceed inductively. Case $m=1$ is trivial. Consider the polynomial $P_{1}(t)=a_{1} t^{n_{1}}$, where $\left|a_{1}\right| \geq \delta$. There exists $l_{1} \in \mathbb{N}$ such that $p n_{1} \geq 1$ for any $n_{1} \geq l_{1}$. Hence

$$
\int_{0}^{1} p n_{1}\left|P_{1}(t)\right|^{p} d t=\frac{p n_{1}}{p n_{1}+1}\left|a_{1}\right|^{p} \geq \frac{1}{2} \delta^{p}=: c_{1} .
$$

For the case $m=2$ firstly we choose a suitable natural number $l_{2}$. To do this let us observe that there exists $\alpha>0$ such that $e^{-\frac{p \alpha(\eta-1)}{q}}<\frac{\delta}{10}$. Select $l_{2} \in \mathbb{N}$ such that $\frac{p n_{1}}{p n_{1}+1} \geq \frac{9}{10}$ and $\left(1-\frac{\alpha}{n_{1}}\right)^{\frac{p n_{1}+1}{q}} \geq \frac{5}{9} e^{-\frac{p \alpha}{q}}$ for any $n_{1}>l_{2}$. Then we may consider the polynomial $P_{2}(t)=a_{1} t^{n_{1}}+a_{2} t^{n_{2}}$, where $\eta<\frac{n_{2}}{n_{1}}<2, n_{1}>l_{2}$ and $\delta \leq \max \left\{\left|a_{1}\right|,\left|a_{2}\right|\right\} \leq 1$. Assume first that $\left|a_{1}\right|<\left(\frac{2}{5} \delta\right)^{\frac{q}{p}}$ and so $\left|a_{2}\right| \geq \delta$. We may estimate

$$
\begin{aligned}
\left(\int_{0}^{1} p n_{2}\left|P_{2}(t)\right|^{p} d t\right)^{\frac{1}{q}} & \geq\left(\frac{p n_{2}}{p n_{2}+1}\right)^{\frac{1}{q}}\left|a_{2}\right|^{\frac{p}{q}} \\
& -\left(\frac{p n_{2}}{p n_{1}+1}\right)^{\frac{1}{q}}\left|a_{1}\right|^{\frac{p}{q}} \geq \frac{9}{10} \delta-2\left(\frac{p n_{1}}{p n_{1}+1}\right)^{\frac{1}{q}}\left|a_{1}\right|^{\frac{p}{q}} \\
& \geq \frac{9}{10} \delta-\frac{4}{5} \delta=\frac{\delta}{10} .
\end{aligned}
$$

If $\left|a_{1}\right| \geq\left(\frac{2}{5} \delta\right)^{\frac{q}{p}}$, then we obtain what follows

$$
\begin{aligned}
\left(\int_{0}^{1} p n_{2}\left|P_{2}(t)\right|^{p} d t\right)^{\frac{1}{q}} & \geq\left(\int_{0}^{1-\frac{\alpha}{n_{1}}} p n_{2}\left|P_{2}(t)\right|^{p} d t\right)^{\frac{1}{q}} \\
& \geq\left(\int_{0}^{1-\frac{\alpha}{n_{1}}} p n_{2}\left|a_{1}\right|^{p} t^{p n_{1}} d t\right)^{\frac{1}{q}} \\
& -\left(\int_{0}^{1-\frac{\alpha}{n_{1}}} p n_{2}\left|a_{2}\right|^{p} t^{p n_{2}} d t\right)^{\frac{1}{q}} \\
& \geq\left(\frac{p n_{2}}{p n_{1}+1}\right)^{\frac{1}{q}}\left(1-\frac{\alpha}{n_{1}}\right)^{\frac{p n_{1}+1}{q}}\left|a_{1}\right|^{\frac{p}{q}}
\end{aligned}
$$




$$
\begin{aligned}
& -\left(\frac{p n_{2}}{p n_{2}+1}\right)^{\frac{1}{q}}\left(1-\frac{\alpha}{n_{1}}\right)^{\frac{p n_{2}+1}{q}}\left|a_{2}\right|^{\frac{p}{q}} \\
\geq & \eta^{\frac{1}{q}} \frac{p n_{1}}{p n_{1}+1}\left(1-\frac{\alpha}{n_{1}}\right)^{\frac{p n_{1}+1}{q}}\left|a_{1}\right|^{\frac{p}{q}}-\left(1-\frac{\alpha}{n_{1}}\right)^{\frac{p n_{2}+1}{q}} \\
\geq & \frac{\delta}{5} e^{-\frac{p \alpha}{q}}-e^{-\frac{p \eta \alpha}{q}}=e^{-\frac{p \alpha}{q}}\left(\frac{\delta}{5}-e^{-\frac{p \alpha(\eta-1)}{q}}\right) \geq \frac{\delta}{10} e^{-\frac{p \alpha}{q}} .
\end{aligned}
$$

Now it is enough to define $c_{2}:=\frac{\delta}{10} e^{-\frac{p \alpha}{q}}$.

Proceeding inductively assume that the statement of the lemma holds true for $m-1$ with constants $c_{m-1} \in(0, \delta)$ and $l_{m-1} \in \mathbb{N}$. There exists $\beta>0$ such that $e^{-\frac{p \beta(\eta-1)}{q}}<$ $\frac{9}{10} \frac{c_{m-1}}{(m-1) 2^{2 m-1}}$. Then select an integer $l_{m} \geq l_{m-1}$ such that $\frac{p n_{1}}{p n_{1}+1}\left(1-\frac{\beta}{n_{1}}\right)^{\frac{p n_{1}+1}{q}} \geq$ $\frac{9}{10} e^{-\frac{p \beta}{q}}$ for any $n_{1}>l_{m}$. Consider the polynomial $P_{m}(t)=\sum_{j=1}^{m} a_{j} t^{n_{j}}$, where $\delta \leq \max _{1 \leq j \leq m}\left|a_{j}\right| \leq 1$ and $\eta<\frac{n_{j+1}}{n_{j}}<2$ for $j=1, \ldots, m-1$ with $n_{1}>l_{m}$. Since the statement of the lemma holds true for $m-1$ with the constant $c_{m-1}$, this means in particular that if $\max _{2 \leq j \leq m}\left|a_{j}\right| \geq \delta$, then

$$
\int_{0}^{1} p n_{m}\left|\sum_{j=2}^{m} a_{j} t^{n_{j}}\right|^{p} d t \geq c_{m-1} .
$$

If $\left|a_{1}\right|<\left(\frac{c_{m-1}}{2^{m}}\right)^{\frac{q}{p}}<\delta$, then $\max _{2 \leq j \leq m}\left|a_{j}\right| \geq \delta$ and from the induction hypothesis we may estimate

$$
\begin{aligned}
\left(\int_{0}^{1} p n_{m}\left|P_{m}(t)\right|^{p} d t\right)^{\frac{1}{q}} & =\left(\int_{0}^{1} p n_{m}\left|\sum_{j=1}^{m} a_{j} t^{n_{j}}\right|^{p} d t\right)^{\frac{1}{q}} \\
& \geq\left(\int_{0}^{1} p n_{m}\left|\sum_{j=2}^{m} a_{j} t^{n_{j}}\right|^{p} d t\right)^{\frac{1}{q}} \\
& -\left(\int_{0}^{1} p n_{m}\left|a_{1}\right|^{p} t^{p n_{1}} d t\right)^{\frac{1}{q}} \\
\geq & c_{m-1}^{\frac{1}{q}}-\left(\frac{p n_{m}}{p n_{1}+1}\right)^{\frac{1}{q}}\left|a_{1}\right|^{\frac{p}{q}} \\
\geq & c_{m-1}-2^{m-1}\left(\frac{p n_{1}}{p n_{1}+1}\right)^{\frac{1}{q}}\left|a_{1}\right|^{\frac{p}{q}} \\
\geq & \frac{1}{2} c_{m-1}
\end{aligned}
$$


If $\left|a_{1}\right| \geq\left(\frac{c_{m-1}}{2^{m}}\right)^{\frac{q}{p}}$, then we obtain

$$
\begin{aligned}
& \left(\int_{0}^{1} p n_{m}\left|P_{m}(t)\right|^{p} d t\right)^{\frac{1}{q}} \geq\left(\int_{0}^{1-\frac{\beta}{n_{1}}} p n_{m}\left|\sum_{j=1}^{m} a_{j} t^{n_{j}}\right|^{p} d t\right)^{\frac{1}{q}} \\
& \geq\left(\int_{0}^{1-\frac{\beta}{n_{1}}} p n_{m}\left|a_{1}\right|^{p} t^{p n_{1}} d t\right)^{\frac{1}{q}}-\left(\sum_{j=2}^{m} \int_{0}^{1-\frac{\beta}{n_{1}}} p n_{m}\left|a_{j}\right|^{p} t^{p n_{j}} d t\right)^{\frac{1}{q}} \\
& \geq\left(\int_{0}^{1-\frac{\beta}{n_{1}}} p n_{m}\left|a_{1}\right|^{p} t^{p n_{1}} d t\right)^{\frac{1}{q}}-\sum_{j=2}^{m}\left(\int_{0}^{1-\frac{\beta}{n_{1}}} p n_{m}\left|a_{j}\right|^{p} t^{p n_{j}} d t\right)^{\frac{1}{q}} \\
& \geq\left(\frac{p n_{m}}{p n_{1}+1}\right)^{\frac{1}{q}}\left(1-\frac{\beta}{n_{1}}\right)^{\frac{p n_{1}+1}{q}}\left|a_{1}\right|^{\frac{p}{q}}-\sum_{j=2}^{m}\left(\frac{p n_{m}}{p n_{j}+1}\right)^{\frac{1}{q}}\left(1-\frac{\beta}{n_{1}}\right)^{\frac{p n_{j}+1}{q}}\left|a_{j}\right|^{\frac{p}{q}} \\
& \geq\left(\frac{p n_{1}}{p n_{1}+1}\right)^{\frac{1}{q}}\left(1-\frac{\beta}{n_{1}}\right)^{\frac{p n_{1}+1}{q}}\left|a_{1}\right|^{\frac{p}{q}}-\sum_{j=2}^{m}\left(\frac{2^{m-j} p n_{j}}{p n_{j}+1}\right)^{\frac{1}{q}}\left(1-\frac{\beta}{n_{1}}\right)^{\frac{p n_{j}+1}{q}} \\
& \geq \frac{p n_{1}}{p n_{1}+1}\left(1-\frac{\beta}{n_{1}}\right)^{\frac{p n_{1}+1}{q}}\left|a_{1}\right|^{\frac{p}{q}}-\sum_{j=2}^{m} 2^{m-j} e^{-\frac{\beta \eta^{j}-1}{q}} \\
& \geq \frac{9}{10} e^{-\frac{p \beta}{q}} \frac{c_{m-1}}{2^{m}}-(m-1) 2^{m-2} e^{-\frac{p \beta \eta}{q}} \\
& \geq e^{-\frac{p \beta}{q}}\left(\frac{9}{10} \frac{c_{m-1}}{2^{m}}-(m-1) 2^{m-2} e^{-\frac{p \beta(\eta-1)}{q}}\right) \geq 0.9 e^{-\frac{p \beta}{q}} \frac{c_{m-1}}{2^{m+1}} .
\end{aligned}
$$

Now from the above inequalities, we may define suitably the constant $c_{m}$ and this completes the proof.

Corollary 3.7 Let $p>0, K \in \mathbb{N}$. There exist constants $c_{K} \in\left(0, \frac{1}{2}\right), C_{K}>1$ and $l_{K} \in \mathbb{N}$ such that for any lacunary $K$-summing polynomial $Q$ of degree $N \geq l_{K}$ the following inequalities hold

$$
c_{K} \leq \int_{0}^{1} p N|Q(z t)|^{p} d t \leq C_{K}, \quad z \in \partial \Omega .
$$

Proof Let $C_{K}:=2 K^{\max \{p, 1\}}$ and $c_{K} \in\left(0, \frac{1}{2}\right), l_{K} \in \mathbb{N}$ be constants from Lemma 3.6 with $m:=K, \eta:=\sqrt[K]{2}$ and $\delta:=\frac{1}{2}$. Let $\sum_{j=1}^{K} u_{k}$ be the homogeneous expansion of $Q$, where each $u_{j}$ is a homogeneous polynomial of degree $n_{j} \in \mathbb{N}$. Observe that since $Q$ is a lacunary $K$-summing polynomial of degree $N \geq l_{K}$, for $z \in \partial \Omega$ polynomial $Q$ satisfies Lemma 3.6 with $a_{j}:=u_{j}(z)$, i.e.

$$
\int_{0}^{1} p N|Q(z t)|^{p} d t=\int_{0}^{1} p N\left|\sum_{j=1}^{K} u_{j}(z) t^{n_{j}}\right|^{p} d t \geq c_{K} .
$$


To prove the second inequality let $q=\max \{p, 1\}$. Then we may estimate

$$
\begin{aligned}
\left(\int_{0}^{1} p N|Q(z t)|^{p} d t\right)^{\frac{1}{q}} & =\left(\int_{0}^{1} p N\left|\sum_{j=1}^{K} u_{j}(z) t^{n_{j}}\right|^{p} d t\right)^{\frac{1}{q}} \\
& \leq \sum_{j=1}^{K}\left(\int_{0}^{1} p N\left|u_{j}(z)\right|^{p} t^{p n_{j}} d t\right)^{\frac{1}{q}} \\
& \leq \sum_{j=1}^{K}\left(\frac{p N}{p n_{j}+1}\right)^{\frac{1}{q}} \leq K\left(\frac{p N}{\frac{1}{2} p N+1}\right)^{\frac{1}{q}} \leq K \sqrt[q]{2} .
\end{aligned}
$$

Hence $\int_{0}^{1} p N|Q(z t)|^{p} d t \leq C_{K}$.

Lemma 3.8 Let $p>0, \varepsilon>0$ and $m \in \mathbb{N}$. There exists $N \in \mathbb{N}$ such that for $n \in \mathbb{N}$ if $n \geq N$, then

$$
(2 p n)^{\frac{1}{p}} \frac{(p n+p m+1)^{\frac{1}{p}}-(p n+1)^{\frac{1}{p}}}{(p n+p m+1)^{\frac{1}{p}}(p n+1)^{\frac{1}{p}}}<\varepsilon .
$$

Proof Select $k \in \mathbb{N}$ such that $k \geq \frac{1}{p}$, so $\frac{1}{p k} \leq 1$. Then by triangle inequality we may estimate

$$
\begin{aligned}
\left((p m+p n+1)^{\frac{1}{p k}}\right)^{k} & \leq\left((p m)^{\frac{1}{p k}}+(p n+1)^{\frac{1}{p k}}\right)^{k}=\sum_{j=0}^{k}\left(\begin{array}{c}
k \\
j
\end{array}\right)(p m)^{\frac{j}{p k}}(p n+1)^{\frac{k-j}{p k}} \\
& =(p n+1)^{\frac{1}{p}}+\sum_{j=1}^{k}\left(\begin{array}{c}
k \\
j
\end{array}\right)(p m)^{\frac{j}{p k}}(p n+1)^{\frac{k-j}{p k}} .
\end{aligned}
$$

Therefore

$$
\begin{aligned}
& (2 p n)^{\frac{1}{p}} \frac{(p n+p m+1)^{\frac{1}{p}}-(p n+1)^{\frac{1}{p}}}{(p n+p m+1)^{\frac{1}{p}}(p n+1)^{\frac{1}{p}}} \leq(2 p n)^{\frac{1}{p}} \frac{\sum_{j=1}^{k}\left(\begin{array}{c}
k \\
j
\end{array}\right)(p m)^{\frac{j}{p k}}(p n+1)^{\frac{k-j}{p k}}}{(p n+p m+1)^{\frac{1}{p}}(p n+1)^{\frac{1}{p}}} \\
& =\frac{(2 p n)^{\frac{1}{p}}}{(p n+p m+1)^{\frac{1}{p}}} \sum_{j=1}^{k}\left(\begin{array}{l}
k \\
j
\end{array}\right)(p m)^{\frac{j}{p k}}(p n+1)^{\frac{-j}{p k}} \\
& \underset{n \rightarrow \infty}{\longrightarrow} 0 \text {. }
\end{aligned}
$$


Hence there exists $N \in \mathbb{N}$ such that for any $n \geq N$ we have

$$
(2 p n)^{\frac{1}{p}} \frac{(p n+p m+1)^{\frac{1}{p}}-(p n+1)^{\frac{1}{p}}}{(p n+p m+1)^{\frac{1}{p}}(p n+1)^{\frac{1}{p}}}<\varepsilon .
$$

Lemma 3.9 Let $p>0, K \in \mathbb{N}$ and $\varepsilon>0$. For every polynomial $f$ there exists $N_{1} \in \mathbb{N}$ such that if $Q$ is a $K$-summing polynomial of degree $N \geq N_{1}$, then

$$
\left|\mathcal{S}^{p}(\sqrt[p]{p N} f Q)-f \mathcal{S}^{p}(\sqrt[p]{p N} Q)\right|<\varepsilon \text { in } \bar{\Omega}
$$

Proof Let $\sum_{i=1}^{L} v_{i}$ be the homogeneous expansion of $f$ with $\operatorname{deg}\left(v_{i}\right)=m_{i} \in \mathbb{N}, i=$ $1,2, \ldots, L$. By Cauchy estimates in the expansion of $f$, there exists a constant $C$ such that $\left\|v_{i}\right\|_{\Omega}<C\|f\|_{\Omega}, i=1,2, \ldots, L$. Lemma 3.8 implies that there exists $N_{1} \in \mathbb{N}$ such that if $N \geq N_{1}$, then for each $m_{i}$ and all $n \in\left[\frac{1}{2} N, N\right]$ we have

$$
\begin{aligned}
& (p N)^{\frac{1}{p}} \frac{\left(p n+p m_{i}+1\right)^{\frac{1}{p}}-(p n+1)^{\frac{1}{p}}}{\left(p n+p m_{i}+1\right)^{\frac{1}{p}}(p n+1)^{\frac{1}{p}}} \\
& \leq(2 p n)^{\frac{1}{p}} \frac{\left(p n+p m_{i}+1\right)^{\frac{1}{p}}-(p n+1)^{\frac{1}{p}}}{\left(p n+p m_{i}+1\right)^{\frac{1}{p}}(p n+1)^{\frac{1}{p}}}<\frac{\varepsilon}{C K L\|f\|_{\Omega}} .
\end{aligned}
$$

Let $\sum_{j=1}^{K} u_{j}$ be the homogeneous expansion of $Q$ with $\operatorname{deg}\left(u_{j}\right)=n_{j} \in \mathbb{N}$, $j=1,2, \ldots, K$. If $Q$ is a $K$-summing polynomial of degree $N \geq N_{1}$, then $n_{j}$ $\in\left[\frac{1}{2} N, N\right], j=1,2, \ldots, K$. Hence by (7), in $\bar{\Omega}$ we obtain what follows

$$
\begin{aligned}
\left|\mathcal{S}^{p}(\sqrt[p]{p N} f Q)-f \mathcal{S}^{p}(\sqrt[p]{p N} Q)\right|=\mid \mathcal{S}^{p}\left(\sqrt[p]{p N} \sum_{i=1}^{L} \sum_{j=1}^{K} v_{i} u_{j}\right) \\
\quad-\sum_{i=1}^{L} v_{i} \mathcal{S}^{p}\left(\sqrt[p]{p N} \sum_{j=1}^{K} u_{j}\right) \mid \\
=\left|\sqrt[p]{p N} \sum_{i=1}^{L} \sum_{j=1}^{K} v_{i} u_{j}\left(\frac{1}{\sqrt[p]{p\left(m_{i}+n_{j}\right)+1}}-\frac{1}{\sqrt[p]{p n_{j}+1}}\right)\right|
\end{aligned}
$$




$$
\begin{aligned}
& \leq \sum_{i=1}^{L} \sum_{j=1}^{K}\left|v_{i}\right|\left|u_{j}\right|\left|\frac{\sqrt[p]{p N}\left(\sqrt[p]{p n_{j}+1}-\sqrt[p]{p\left(m_{i}+n_{j}\right)+1}\right)}{\sqrt[p]{p\left(m_{i}+n_{j}\right)+1} \sqrt[p]{p n_{j}+1}}\right| \\
& <\sum_{i=1}^{L} \sum_{j=1}^{K}\left|v_{i}\right|\left|u_{j}\right| \frac{\varepsilon}{C K L\|f\|_{\Omega}}<\varepsilon
\end{aligned}
$$

This completes the proof.

Remark 3.10 Let $p>0$ and $K \in \mathbb{N}$. If $Q$ is a $K$-summing polynomial, then for $z \in \partial \Omega$

$$
\left|\mathcal{S}^{p}(\sqrt[p]{p \operatorname{deg}(Q)} Q)(z)\right|<K \sqrt[p]{2}
$$

Proof Let $\sum_{j=1}^{K} u_{j}$ be the homogeneous expansion of $Q$ with $\operatorname{deg}\left(u_{j}\right)=n_{j} \in$ $\mathbb{N}, j=1,2, \ldots, K$. If $Q$ is a $K$-summing polynomial, then $\frac{1}{2} \operatorname{deg}(Q) \leq n_{j} \leq$ $\operatorname{deg}(Q), \quad j=1,2, \ldots, K$. By properties of $K$-summing polynomials, we obtain the required estimate on $\partial \Omega$

$$
\begin{aligned}
\left|\mathcal{S}^{p}(\sqrt[p]{p \operatorname{deg}(Q)} Q)\right| & =\left|\mathcal{S}^{p}\left(\sqrt[p]{p \operatorname{deg}(Q)} \sum_{j=1}^{K} u_{j}\right)\right|=\left|\sum_{j=1}^{K} \sqrt[p]{\frac{p \operatorname{deg}(Q)}{p n_{j}+1}} u_{j}\right| \\
& \leq \sqrt[p]{\frac{p \operatorname{deg}(Q)}{\frac{1}{2} p \operatorname{deg}(Q)+1}} \sum_{j=1}^{K}\left|u_{j}\right| \quad<K \sqrt[p]{2} .
\end{aligned}
$$

\section{Radon Inversion Problem}

As we saw in the previous section, lacunary $K$-summing polynomials possess some interesting properties. Herein, we shall show how we can use them to solve by induction the Radon inversion problem with given a strictly positive, continuous function $\Phi$. First of all, in Theorem 4.1 we construct generators which at each induction step allow us to come closer to $\Phi$ by a constant fraction of what is still missing. Moreover, the operator $\mathcal{R}^{p}$ is "almost additive" in the space of generators with respect to a given $\varepsilon>0$. From this, one concludes that the sum of constructed generators converges in $\mathcal{H} \mathcal{R}^{p}(\Omega)$ and solves the Radon inversion problem with $\Phi$ as we will see it in Theorem 4.2.

Theorem 4.1 Let $p>0$. There exist $\theta \in(0,1)$ and $\kappa>0$ such that if $\Psi$ is a strictly positive continuous function on $\partial \Omega$ and $\varepsilon, N_{0} \in \mathbb{N}, M_{0}>0$, then there exists $a$ polynomial $F$ of order greater than $N_{0}$ with the following properties

(c1) $\theta \Psi<\mathcal{R}^{p}(F)<\Psi$ on $\partial \Omega$

(c2) $\mathcal{R}^{p}(\phi)+\mathcal{R}^{p}(F)-\varepsilon \leq \mathcal{R}^{p}(\phi+F) \leq \mathcal{R}^{p}(\phi)+\mathcal{R}^{p}(F)+\varepsilon$ on $\partial \Omega$ for any $\phi \in \mathcal{C}_{M_{0}}(\bar{\Omega})$ 
(c3) $\left|\mathcal{S}^{p}(F)\right|<\kappa(\Psi)^{\frac{1}{p}}$ on $\partial \Omega$.

Proof Take $N=N(\partial \Omega), K=K(\partial \Omega)$ and $N_{K} \in \mathbb{N}$ from Theorem 2.2 and Corollary 3.3, respectively. For such $K$ we may choose $c_{K} \in\left(0, \frac{1}{2}\right), C_{K}>1$ and $l_{K} \in \mathbb{N}$ from Corollary 3.7. We shall show that $\theta:=\frac{c_{K}}{8 N C_{K}}$ and $\kappa:=\frac{N(K+1)}{\sqrt[p]{C_{K}}}$ satisfy the statement of Theorem 4.1.

First of all, by Theorem 2.2, there exist polynomials $f_{1}, \ldots, f_{N}$ such that

$$
\frac{\Psi}{2 N}<\max _{1 \leq m \leq N}\left|f_{m}\right|^{p}<\frac{\Psi}{N} \text { on } \partial \Omega \text {. }
$$

Let $f_{0} \equiv 0, \widehat{\varepsilon}:=\min \left\{\varepsilon, \theta \inf _{z \in \partial \Omega} \Psi(z)\right\}$ and $\alpha:=\frac{1}{\sqrt[p]{C_{K}}} \inf _{z \in \partial \Omega}(\Psi(z))^{\frac{1}{p}}$. Then by Lemma 3.5 and Lemma 3.9, there exists an integer $\bar{N} \geq \max \left\{2 N_{0}, l_{K}, N_{K}\right\}$ such that for each $m=0,1, \ldots, N$ any $K$-summing polynomial $Q$ of degree $n \geq \bar{N}$ satisfies the following inequalities for $z \in \partial \Omega$

$$
\begin{aligned}
& \left|\mathcal{S}^{p}\left(\sqrt[p]{p N} f_{m} Q\right)(z)-f_{m} \mathcal{S}^{p}(\sqrt[p]{p N} Q)(z)\right|<\frac{\alpha}{N}, \\
& \left|f_{m}(z)\right|^{p} \mathcal{R}^{p}(Q)(z)-\frac{\widehat{\varepsilon}}{N} \leq \mathcal{R}^{p}\left(f_{m} Q\right)(z) \leq\left|f_{m}(z)\right|^{p} \mathcal{R}^{p}(Q)(z)+\frac{\widehat{\varepsilon}}{N} \\
& c_{K} \leq \int_{0}^{1} p \operatorname{deg}(Q)|Q(z t)|^{p} d t \leq C_{K}
\end{aligned}
$$

Inductively we shall choose integers $n_{0}, n_{1}, \ldots, n_{N}$ and construct lacunary $K$ summing polynomials $Q_{0}, Q_{1}, \ldots, Q_{N}$ of orders at least $n_{0}, n_{1}, \ldots, n_{N}$, respectively, such that for $m=0,1, \ldots, N$ and for any $\phi \in \mathcal{C}_{M_{0}}(\bar{\Omega})$ we have on $\partial \Omega$

$$
\begin{aligned}
& \mathcal{R}^{p}(\phi)+\mathcal{R}^{p}\left(\sum_{j=0}^{m} f_{j} Q_{j}\right)-\frac{m \widehat{\varepsilon}}{N} \\
& \leq \mathcal{R}^{p}\left(\phi+\sum_{j=0}^{m} f_{j} Q_{j}\right) \leq \mathcal{R}^{p}(\phi)+\mathcal{R}^{p}\left(\sum_{j=0}^{m} f_{j} Q_{j}\right)+\frac{m \widehat{\varepsilon}}{N} .
\end{aligned}
$$

Let $n_{0}:=0$ and $Q_{0} \equiv 1$ in $\bar{\Omega}$. For $m=1$, we begin by selecting an integer $n_{1} \geq \bar{N}$ from Lemma 3.4 applied with $\varepsilon:=\frac{\widehat{\varepsilon}}{N}, M:=M_{0}, f:=f_{1}$. Then by Corollary 3.3, there exists a lacunary $K$-summing polynomial $Q_{1}$ of order greater than $n_{1}$ such that $\mathcal{R}^{p}(\phi)+\mathcal{R}^{p}\left(f_{1} Q_{1}\right)-\frac{\widehat{\varepsilon}}{N} \leq \mathcal{R}^{p}\left(\phi+f_{1} Q_{1}\right) \leq \mathcal{R}^{p}(\phi)+\mathcal{R}^{p}\left(f_{1} Q_{1}\right)+\frac{\widehat{\varepsilon}}{N}$ on $\partial \Omega$.

Proceeding inductively, assume that we have already chosen $n_{0}, n_{1}, \ldots, n_{m}$ and constructed lacunary $K$-summing polynomials $Q_{0}, Q_{1}, \ldots, Q_{m}$ of orders greater than $n_{1}, n_{2}, \ldots, n_{m}$, respectively, with desired properties, $0<m<N$. Then again apply 
Lemma 3.4 with

$$
\varepsilon:=\frac{\widehat{\varepsilon}}{2 N}, \quad M:=\sup _{z \in \partial \Omega}\left(|\phi(z)|+\left|\sum_{j=0}^{m} f_{j}(z) Q_{j}(z)\right|\right), \quad f:=f_{m+1}
$$

to obtain an integer $n_{m+1} \geq \bar{N}$. Then Corollary 3.3 produces a lacunary $K$-summing polynomial $Q_{m+1}$ of order greater than $n_{m+1}$ such that on $\partial \Omega$ the following inequalities are satisfied

$$
\begin{aligned}
\mathcal{R}^{p}\left(\phi+\sum_{j=0}^{m+1} f_{j} Q_{j}\right) & \geq \mathcal{R}^{p}\left(\phi+\sum_{j=0}^{m} f_{j} Q_{j}\right)+\mathcal{R}^{p}\left(f_{m+1} Q_{m+1}\right)-\frac{\widehat{\varepsilon}}{2 N} \\
& \geq \mathcal{R}^{p}(\phi)+\mathcal{R}^{p}\left(\sum_{j=0}^{m} f_{j} Q_{j}\right)+\mathcal{R}^{p}\left(f_{m+1} Q_{m+1}\right)-\frac{m \widehat{\varepsilon}}{N}-\frac{\widehat{\varepsilon}}{2 N} \\
& \geq \mathcal{R}^{p}(\phi)+\mathcal{R}^{p}\left(\sum_{j=0}^{m+1} f_{j} Q_{j}\right)-\frac{(m+1) \widehat{\varepsilon}}{N}, \\
\mathcal{R}^{p}\left(\phi+\sum_{j=0}^{m+1} f_{j} Q_{j}\right) & \leq \mathcal{R}^{p}\left(\phi+\sum_{j=0}^{m} f_{j} Q_{j}\right)+\mathcal{R}^{p}\left(f_{m+1} Q_{m+1}\right)-\frac{\widehat{\varepsilon}}{2 N} \\
& \leq \mathcal{R}^{p}(\phi)+\mathcal{R}^{p}\left(\sum_{j=0}^{m} f_{j} Q_{j}\right)+\mathcal{R}^{p}\left(f_{m+1} Q_{m+1}\right)+\frac{m \widehat{\varepsilon}}{N}+\frac{\widehat{\varepsilon}}{2 N} \\
& \leq \mathcal{R}^{p}(\phi)+\mathcal{R}^{p}\left(\sum_{j=0}^{m+1} f_{j} Q_{j}\right)+\frac{(m+1) \widehat{\varepsilon}}{N} .
\end{aligned}
$$

Define the polynomial $F(z)=\sum_{m=1}^{N} \sqrt[p]{\frac{p \operatorname{deg}\left(Q_{m}\right)}{K C_{K}}} f_{m}(z) Q_{m}(z)$. Observe that by (12), the polynomial $F$ satisfies condition ( $(2)$. For each $z \in \partial \Omega$ one may choose an index $m_{z} \in\{1, \ldots, N\}$ for which $\left|f_{m_{z}}(z)\right|^{p}=\max _{1 \leq m \leq N}\left|f_{m}(z)\right|^{p}>\frac{\Psi(z)}{2 N}$. Then for $z \in \partial \Omega$ we obtain

$$
\begin{aligned}
\mathcal{R}^{p}(F)(z) & =\int_{0}^{1}\left|\sum_{m=1}^{N} \sqrt[p]{\frac{p \operatorname{deg}\left(Q_{m}\right)}{2 C_{K}}} f_{m}(z t) Q_{m}(z t)\right|^{p} d t \\
& \stackrel{(12)}{\geq} \sum_{m=1}^{N}\left|f_{m}(z)\right|^{p} \frac{p \operatorname{deg}\left(Q_{m}\right)}{2 C_{K}} \int_{0}^{1}\left|Q_{m}(z t)\right|^{p} d t-\frac{c_{K}}{8 N C_{K}} \inf _{z \in \partial \Omega} \Psi(z) \\
& \geq\left|f_{m_{z}}(z)\right|^{p} \frac{p \operatorname{deg}\left(Q_{m_{z}}\right)}{2 C_{K}} \int_{0}^{1}\left|Q_{m_{z}}(z t)\right|^{p} d t-\frac{c_{K}}{8 N C_{K}} \inf _{z \in \partial \Omega} \Psi(z)
\end{aligned}
$$




$$
\begin{aligned}
& \stackrel{(11)}{\geq} \frac{c_{K}}{2 C_{K}}\left|f_{m_{z}}(z)\right|^{p}-\frac{c_{K}}{8 N C_{K}} \inf _{z \in \partial \Omega} \Psi(z) \stackrel{(8)}{>} \frac{c_{K}}{4 N C_{K}} \Psi(z)-\frac{c_{K}}{8 N C_{K}} \Psi(z) \\
& =\frac{c_{K}}{8 N C_{K}} \Psi(z)=\theta \Psi(z) .
\end{aligned}
$$

Moreover,

$$
\begin{aligned}
\mathcal{R}^{p}(F)(z) & =\int_{0}^{1}\left|\sum_{m=1}^{N} \sqrt[p]{\frac{p \operatorname{deg}\left(Q_{m}\right)}{2 C_{K}}} f_{m}(z t) Q_{m}(z t)\right|^{p} d t \\
& \stackrel{(12)}{\leq} \sum_{m=1}^{N}\left|f_{m}(z)\right|^{p} \frac{p \operatorname{deg}\left(Q_{m}\right)}{2 C_{K}} \int_{0}^{1}\left|Q_{m}(z t)\right|^{p} d t+\frac{c_{K}}{8 N C_{K}} \inf _{z \in \partial \Omega} \Psi(z) \\
& \stackrel{(8)}{<} \frac{\Psi(z)}{2 C_{K}} C_{K}+\frac{1}{2} \Psi(z)=\Psi(z) .
\end{aligned}
$$

Since any $Q_{m}$ satisfies (9), for $z \in \partial \Omega$ we have the following estimate

$$
\begin{aligned}
\left|\mathcal{S}^{p}(F)(z)\right| & =\left|\mathcal{S}^{p}\left(\sum_{m=1}^{N} \sqrt[p]{\frac{p \operatorname{deg}\left(Q_{m}\right)}{2 C_{K}}} f_{m} Q_{m}\right)(z)\right| \\
& \leq \frac{1}{\sqrt[p]{2 C_{K}}} \sum_{m=1}^{N}\left|\mathcal{S}^{p}\left(\sqrt[p]{p \operatorname{deg}\left(Q_{m}\right)} f_{m} Q_{m}\right)(z)\right| \\
& \left(\stackrel{9}{<} \frac{1}{\sqrt[p]{2 C_{K}}} \sum_{m=1}^{N}\left|f_{m}(z)\right|\left|\mathcal{S}^{p}\left(\sqrt[p]{p \operatorname{deg}\left(Q_{m}\right)} Q_{m}\right)(z)\right|+\frac{N}{\sqrt[p]{C_{K}}} \inf _{z \in \partial \Omega}(\Psi(z))^{\frac{1}{p}}\right. \\
& \leq \frac{N K \sqrt[p]{2}}{\sqrt[p]{2 C_{K}}}\left|f_{m_{z}}(z)\right|+\frac{N}{\sqrt[p]{C_{K}}}(\Psi(z))^{\frac{1}{p}}<\kappa(\Psi(z))^{\frac{1}{p}} .
\end{aligned}
$$

This completes the proof.

Once we proved Theorem 4.1, we are ready to give the first construction of a solution $G$ to the Radon inversion problem such that $\mathcal{S}^{p}(G)$ is continuous up to the boundary of the domain.

Theorem 4.2 Let $p>0$ and $\Phi$ be a strictly positive, continuous function on $\partial \Omega$. There exists a function $G \in \mathcal{O}(\Omega)$ such that

$$
\mathcal{R}^{p}(G)(z)=\Phi(z) \text { for } z \in \partial \Omega \text {. }
$$

Moreover, $\mathcal{S}^{p}(G) \in \mathcal{C}(\bar{\Omega})$. 
Proof Take $\theta \in(0,1)$ and $\kappa>0$ from Theorem 4.1. We will show that it is possible to construct inductively a sequence of polynomials $\left\{F_{j}\right\}_{j=0}^{\infty}$ such that

$$
\left(1-\frac{\theta}{2}\right)\left(\Phi(z)-\mathcal{R}^{p}\left(\sum_{i=0}^{j-1} F_{i}\right)(z)\right)>\Phi(z)-\mathcal{R}^{p}\left(\sum_{i=0}^{j} F_{i}\right)(z)>0
$$

and

$$
\left|\mathcal{S}^{p}\left(F_{j}\right)(z)\right|<\kappa\left(1-\frac{\theta}{2}\right)^{\frac{j}{p}}\|\Phi\|_{\partial \Omega}^{\frac{1}{p}}, \quad z \in \partial \Omega, \quad j=1,2, \ldots
$$

Set $F_{0} \equiv 0$ and observe that (14) holds. For $j=1$ we construct a polynomial $F_{1}$ by applying Theorem 4.1 with $\Psi:=\Phi$. Notice that $F_{1}$ satisfies (13) and (14). Then assume that we have constructed polynomials $F_{0}, F_{1}, \ldots, F_{j}$ for $j \geq 1$. Again from Theorem 4.1 applied to

$$
\begin{aligned}
\Psi & :=\frac{3}{4}\left(\Phi-\mathcal{R}^{p}\left(\sum_{i=0}^{j} F_{i}\right)\right), \varepsilon:=\frac{\theta}{4} \inf _{z \in \partial \Omega}\left(\Phi(z)-\mathcal{R}^{p}\left(\sum_{i=0}^{j} F_{i}\right)(z)\right), \\
M_{0} & :=\sup _{z \in \partial \Omega}\left|\sum_{i=0}^{j} F_{i}(z)\right|,
\end{aligned}
$$

there exists a polynomial $F_{j+1}$ such that on $\partial \Omega$

1. $\frac{3}{4} \theta\left(\Phi-\mathcal{R}^{p}\left(\sum_{i=0}^{j} F_{i}\right)\right)<\mathcal{R}^{p}\left(F_{j+1}\right)<\frac{3}{4}\left(\Phi-\mathcal{R}^{p}\left(\sum_{i=0}^{j} F_{i}\right)\right)$

2. $\mathcal{R}^{p}\left(\sum_{i=0}^{j} F_{i}\right)+\mathcal{R}^{p}\left(F_{j+1}\right)-\varepsilon<\mathcal{R}^{p}\left(\sum_{i=0}^{j+1} F_{i}\right)<\mathcal{R}^{p}\left(\sum_{i=0}^{j} F_{i}\right)+\mathcal{R}^{p}\left(F_{j+1}\right)+\varepsilon$

3. $\left|\mathcal{S}^{p}\left(F_{j+1}\right)\right|<\kappa\left(\frac{3}{4}\left(\Phi-\mathcal{R}^{p}\left(\sum_{i=0}^{j} F_{i}\right)\right)\right)^{\frac{1}{p}}$.

Hence on $\partial \Omega$ we have the following estimates

$$
\begin{aligned}
\mathcal{R}^{p}\left(\sum_{i=0}^{j+1} F_{i}\right) & >\mathcal{R}^{p}\left(\sum_{i=0}^{j} F_{i}\right)+\mathcal{R}^{p}\left(F_{j+1}\right)-\varepsilon \\
& >\mathcal{R}^{p}\left(\sum_{i=0}^{j} F_{i}\right)+\frac{3}{4} \theta\left(\Phi-\mathcal{R}^{p}\left(\sum_{i=0}^{j} F_{i}\right)\right)-\varepsilon
\end{aligned}
$$




$$
\begin{aligned}
& >\frac{3}{4} \theta \Phi+\left(1-\frac{3}{4} \theta\right) \mathcal{R}^{p}\left(\sum_{i=0}^{j} F_{i}\right)-\frac{\theta}{4} \inf _{z \in \partial \Omega}\left(\Phi(z)-\mathcal{R}^{p}\left(\sum_{i=0}^{j} F_{i}\right)(z)\right) \\
& >\frac{\theta}{2} \Phi+\left(1-\frac{\theta}{2}\right) \mathcal{R}^{p}\left(\sum_{i=0}^{j} F_{i}\right)
\end{aligned}
$$

and

$$
\begin{aligned}
\mathcal{R}^{p}\left(\sum_{i=0}^{j+1} F_{i}\right)< & \mathcal{R}^{p}\left(\sum_{i=0}^{j} F_{i}\right)+\mathcal{R}^{p}\left(F_{j+1}\right)+\varepsilon \\
< & \mathcal{R}^{p}\left(\sum_{i=0}^{j} F_{i}\right)+\frac{3}{4}\left(\Phi-\mathcal{R}^{p}\left(\sum_{i=0}^{j} F_{i}\right)\right) \\
& +\frac{\theta}{4} \inf _{z \in \partial \Omega}\left(\Phi(z)-\mathcal{R}^{p}\left(\sum_{i=0}^{j} F_{i}\right)(z)\right) \\
\leq & \mathcal{R}^{p}\left(\sum_{i=0}^{j} F_{i}\right)+\frac{3}{4}\left(\Phi-\mathcal{R}^{p}\left(\sum_{i=0}^{j} F_{i}\right)\right)+\frac{1}{4}\left(\Phi-\mathcal{R}^{p}\left(\sum_{i=0}^{j} F_{i}\right)\right) \\
\leq & \Phi .
\end{aligned}
$$

Therefore $\left(1-\frac{\theta}{2}\right)\left(\Phi-\mathcal{R}^{p}\left(\sum_{i=0}^{j} F_{i}\right)\right)>\Phi-\mathcal{R}^{p}\left(\sum_{i=0}^{j+1} F_{i}\right)>0$, which implies that

$$
\left(1-\frac{\theta}{2}\right)^{j+1} \Phi>\Phi-\mathcal{R}^{p}\left(\sum_{i=0}^{j+1} F_{i}\right)>0 \text { on } \partial \Omega .
$$

From this it follows that

$$
\lim _{n \rightarrow \infty} \mathcal{R}^{p}\left(\sum_{j=0}^{n} F_{j}\right)(z)=\Phi(z), \quad z \in \partial \Omega
$$

In addition, $\left|\mathcal{S}^{p}\left(F_{j+1}\right)\right|<\kappa\left(\Phi-\mathcal{R}^{p}\left(\sum_{i=0}^{j} F_{i}\right)\right)^{\frac{1}{p}}<\kappa\left(1-\frac{\theta}{2}\right)^{\frac{j+1}{p}}\|\Phi\|_{\partial \Omega}^{\frac{1}{p}}$ on $\partial \Omega$. 
Let $q=\max \{p, 1\}$ and observe that if $n \geq m$, then

$$
\begin{aligned}
& \sup _{z \in \partial \Omega}\left(\mathcal{R}^{p}\left(\sum_{j=0}^{m} F_{j}-\sum_{j=0}^{n} F_{j}\right)(z)\right)^{\frac{1}{q}} \\
& \quad=\sup _{z \in \partial \Omega}\left(\mathcal{R}^{p}\left(\sum_{j=n+1}^{m} F_{j}\right)(z)\right)^{\frac{1}{q}} \leq \sup _{z \in \partial \Omega}\left(\sum_{j=n+1}^{m} \mathcal{R}^{p}\left(F_{j}\right)(z)\right)^{\frac{1}{q}} \\
& \quad \leq \sup _{z \in \partial \Omega}\left(\sum_{j=n+1}^{m}\left(\Phi(z)-\mathcal{R}^{p}\left(\sum_{i=0}^{j-1} F_{i}\right)(z)\right)^{\frac{1}{q}}\right) \\
& \quad<\sum_{j=n+1}^{m}\left(1-\frac{\theta}{2}\right)^{\frac{j}{q}}\|\Phi\|_{\partial \Omega}^{\frac{1}{q}} \underset{n, m \rightarrow \infty}{\longrightarrow} 0,
\end{aligned}
$$

so $\left\{\sum_{j=0}^{n} F_{j}\right\}$ is a Cauchy sequence in $\mathcal{H R}^{p}(\Omega)$ and by Proposition 2.1, it is convergent in $\mathcal{H R}^{p}(\Omega)$. Hence we may define the function $G:=\sum_{j=0}^{\infty} F_{j}$ and again by Proposition 2.1, $G$ is holomorphic in $\Omega$. Moreover, $\mathcal{R}^{p}\left(\sum_{j=0}^{n} F_{j}\right) \rightrightarrows \mathcal{R}^{p}(G)$ as $n \rightarrow \infty$ on $\partial \Omega$, so $\mathcal{R}^{p}(G)=\lim _{n \rightarrow \infty} \mathcal{R}^{p}\left(\sum_{j=0}^{n} F_{j}\right)=\Phi$.

Since for $z \in \bar{\Omega}$ we have the following estimate

$$
\sum_{j=0}^{\infty}\left|\mathcal{S}^{p}\left(F_{j}\right)(z)\right| \leq \sum_{j=0}^{\infty} \sup _{z \in \partial \Omega}\left|\mathcal{S}^{p}\left(F_{j}\right)(z)\right|<\sum_{j=0}^{\infty} \kappa\left(1-\frac{\theta}{2}\right)^{\frac{j}{p}}\|\Phi\|_{\partial \Omega}^{\frac{1}{p}}<\infty
$$

then $\mathcal{S}^{p}(G)$ is a continuous function in $\bar{\Omega}$. This completes the proof.

\section{Divergent Taylor Series}

In what follows we will show that it is possible to construct a solution $f$ to the Radon inversion problem such that the function $\mathcal{S}^{p}(f)$ in contrast to the solution obtained in Theorem 4.2 has in some sense extremal properties, i.e. every slice function of $\mathcal{S}^{p}(f)$ has a divergent series of Taylor coefficients with every exponent $s<\min \{1, p\}$. Moreover, for $p \leq 1$ function $\mathcal{S}^{p}(f)$ is continuous up to the boundary and for $p \in$ $(1,2]$ is square-integrable on all unit circles $z \partial \mathbb{D}, z \in \partial \Omega$. To prove this we need the following technical lemma.

Lemma 5.1 There exist $\theta \in(0,1)$ and $C>0$ such that if $h \in \mathcal{C}(\partial \Omega)$ is a strictly positive function on $\partial \Omega, g$ is a bounded, continuous function in $\bar{\Omega}, M>0, a, \gamma, \tau \in$ $(0,1)$, then there exist $N \in \mathbb{N}$ and a sequence of orthogonal polynomials $\left\{P_{k}\right\}_{k=0}^{\infty}$ such that

$(w 1) \quad$ ord $\left(P_{0}\right) \geq M$ 
(w2) $\mathcal{R}^{p}\left(P_{k}\right)<$ ah on $\partial \Omega$

(w3) $\operatorname{ord}\left(P_{k}\right)>\operatorname{deg}\left(P_{k-1}\right)$

(w4) $\mathcal{R}^{p}\left(\sum_{j=0}^{k} P_{j}\right)<\mathcal{R}^{p}\left(\sum_{j=0}^{k-1} P_{j}\right)+a\left(h-\mathcal{R}^{p}\left(\sum_{j=0}^{k-1} P_{j}\right)\right)<h$ on $\partial \Omega$

(w5) $\mathcal{R}^{p}\left(\sum_{j=0}^{k} P_{j}\right)>\mathcal{R}^{p}\left(\sum_{j=0}^{k-1} P_{j}\right)+\frac{1}{2} \theta a\left(h-\mathcal{R}^{p}\left(\sum_{j=0}^{k-1} P_{j}\right)\right)$ on $\partial \Omega$

(w6) $\left|\mathcal{R}^{p}\left(\sum_{j=0}^{k} P_{j}\right)-\sum_{j=0}^{k} \mathcal{R}^{p}\left(P_{j}\right)\right|<\tau$ on $\partial \Omega$

(w7) $\left|\mathcal{R}^{p}\left(g+\sum_{j=0}^{k} P_{j}\right)-\sum_{j=0}^{k} \mathcal{R}^{p}\left(P_{j}\right)-\mathcal{R}^{p}(g)(z)\right|<\tau$ on $\partial \Omega$

(w8) $\left|\mathcal{S}^{p}\left(P_{k}\right)\right|<C\left(\mathcal{R}^{p}\left(P_{k}\right)\right)^{\frac{1}{p}}$ on $\partial \Omega$

(w9) $\mathcal{R}^{p}\left(\sum_{j=0}^{N} P_{j}\right)>\gamma h$ on $\partial \Omega$

(w10) if $p \in(0,1]$, then $\left|\mathcal{S}^{p}\left(\sum_{j=0}^{N} P_{j}\right)\right|<\frac{2 C}{\theta} h^{\frac{1}{p}} a^{\frac{1}{p}-1}$ on $\partial \Omega$.

Proof Take constants $\theta \in(0,1)$ and $\kappa>0$ from Theorem 4.1. Let $C:=\frac{\kappa}{\sqrt[p]{\theta}}$. We proceed inductively. For $k=0$ apply Theorem 4.1 with $\Psi:=\frac{1}{2} a h, M_{0}:=$ $\|g\|_{\Omega}, N_{0}:=M, \varepsilon:=\tau$ to obtain a polynomial $P_{0}$ such that $\operatorname{ord}\left(P_{0}\right) \geq M$ and on $\partial \Omega$ the following statements hold

a) $\frac{1}{2} \theta a h<\mathcal{R}^{p}\left(P_{0}\right)<\frac{1}{2} a h$

b) $\mathcal{R}^{p}\left(P_{0}\right)+\mathcal{R}^{p}(g)-\tau<\mathcal{R}^{p}\left(g+P_{0}\right)<\mathcal{R}^{p}\left(P_{0}\right)+\mathcal{R}^{p}(g)-\tau$

c) $\left|\mathcal{S}^{p}\left(P_{0}\right)\right|<\kappa\left(\frac{1}{2} a h\right)^{\frac{1}{p}}<C\left(\mathcal{R}^{p}\left(P_{0}\right)\right)^{\frac{1}{p}}$.

Hence $P_{0}$ satisfies conditions $(w 1)-(w 2)$ and $(w 6)-(w 8)$. Assuming that $\left.b\right)$ holds, there exists $\tau_{0} \in(0,1)$ such that $\left|\mathcal{R}^{p}\left(g+P_{0}\right)-\mathcal{R}^{p}\left(P_{0}\right)-\mathcal{R}^{p}(g)\right|<\tau_{0}<\tau$ on $\partial \Omega$. For $k=1$ we apply Theorem 4.1 to

$$
\begin{aligned}
\Psi & :=\frac{3}{4} a\left(h-\mathcal{R}^{p}\left(P_{0}\right)\right), \quad M_{0}:=\left\|P_{0}\right\|_{\Omega}+\|g\|_{\Omega}, \quad N_{0}:=\operatorname{deg}\left(P_{0}\right)+1, \\
\varepsilon & :=\min \left\{\frac{\theta}{4} \inf _{z \in \partial \Omega} a\left(h(z)-\mathcal{R}^{p}\left(P_{0}\right)(z)\right), \tau-\tau_{0}\right\} .
\end{aligned}
$$

This produces a polynomial $P_{1}$ such that $\operatorname{ord}\left(P_{1}\right)>\operatorname{deg}\left(P_{0}\right)$ and on $\partial \Omega$ the following conditions are satisfied

- $\frac{3}{4} \theta a\left(h-\mathcal{R}^{p}\left(P_{0}\right)\right)<\mathcal{R}^{p}\left(P_{1}\right)<\frac{3}{4} a\left(h-\mathcal{R}^{p}\left(P_{0}\right)\right)<a h$

- $\mathcal{R}^{p}\left(P_{0}\right)+\mathcal{R}^{p}\left(P_{1}\right)-\varepsilon<\mathcal{R}^{p}\left(P_{0}+P_{1}\right)<\mathcal{R}^{p}\left(P_{0}\right)+\mathcal{R}^{p}\left(P_{1}\right)+\varepsilon$

- $\mathcal{R}^{p}\left(g+P_{0}\right)+\mathcal{R}^{p}\left(P_{1}\right)-\varepsilon<\mathcal{R}^{p}\left(g+P_{0}+P_{1}\right)<\mathcal{R}^{p}\left(g+P_{0}\right)+\mathcal{R}^{p}\left(P_{1}\right)+\varepsilon$ 
- $\left|\mathcal{S}^{p}\left(P_{1}\right)\right|<\kappa\left(\frac{3}{4} a\left(h-\mathcal{R}^{p}\left(P_{0}\right)\right)\right)^{\frac{1}{p}}<C\left(\mathcal{R}^{p}\left(P_{1}\right)\right)^{\frac{1}{p}}$.

Then simple calculations give the following estimates on $\partial \Omega$

$$
\mathcal{R}^{p}\left(P_{0}+P_{1}\right)<\mathcal{R}^{p}\left(P_{0}\right)+\mathcal{R}^{p}\left(P_{1}\right)+\varepsilon<\mathcal{R}^{p}\left(P_{0}\right)+a\left(h-\mathcal{R}^{p}\left(P_{0}\right)\right)
$$

and

$$
\mathcal{R}^{p}\left(P_{0}+P_{1}\right)>\mathcal{R}^{p}\left(P_{0}\right)+\mathcal{R}^{p}\left(P_{1}\right)-\varepsilon>\mathcal{R}^{p}\left(P_{0}\right)+\frac{1}{2} \theta a\left(h-\mathcal{R}^{p}\left(P_{0}\right)\right) .
$$

Also since $\varepsilon<\tau$, it is clear that $\mathcal{R}^{p}\left(P_{0}\right)+\mathcal{R}^{p}\left(P_{1}\right)-\tau<\mathcal{R}^{p}\left(P_{0}+P_{1}\right)<\mathcal{R}^{p}\left(P_{0}\right)+$ $\mathcal{R}^{p}\left(P_{1}\right)+\tau$ on $\partial \Omega$. Moreover,

$$
\begin{aligned}
\mathcal{R}^{p}\left(P_{0}+P_{1}+g\right) & <\mathcal{R}^{p}\left(P_{0}+g\right)+\mathcal{R}^{p}\left(P_{1}\right)+\varepsilon \\
& <\mathcal{R}^{p}(g)+\mathcal{R}^{p}\left(P_{0}\right)+\mathcal{R}^{p}\left(P_{1}\right)+\tau_{0}+\varepsilon \\
& =\mathcal{R}^{p}(g)+\mathcal{R}^{p}\left(P_{0}\right)+\mathcal{R}^{p}\left(P_{1}\right)+\tau
\end{aligned}
$$

and similarly $\mathcal{R}^{p}\left(P_{0}+P_{1}+g\right)>\mathcal{R}^{p}(g)+\mathcal{R}^{p}\left(P_{0}\right)+\mathcal{R}^{p}\left(P_{1}\right)-\tau$ on $\partial \Omega$. It follows from the above that $P_{1}$ is orthogonal to $P_{0}$ and has properties $(w 1)-(w 8)$.

We then proceed inductively as follows: Suppose that orthogonal polynomials $P_{0}, P_{1}, \ldots, P_{k}$ have been found such that conditions $(w 1)-(w 8)$ hold. Observe that by statements $(w 6)$ and $(w 7)$ there exist $\tau_{1}, \tau_{2} \in(0, \tau)$ such that

- $\left|\mathcal{R}^{p}\left(\sum_{j=0}^{k} P_{j}\right)-\sum_{j=0}^{k} \mathcal{R}^{p}\left(P_{j}\right)\right|<\tau_{1}<\tau$ on $\partial \Omega$

- $\left|\mathcal{R}^{p}\left(g+\sum_{j=0}^{k} P_{j}\right)-\sum_{j=0}^{k} \mathcal{R}^{p}\left(P_{j}\right)-\mathcal{R}^{p}(g)\right|<\tau_{2}<\tau$ on $\partial \Omega$.

Theorem 4.1 applied to

$$
\begin{aligned}
\Psi & :=\frac{3}{4} a\left(h-\mathcal{R}^{p}\left(\sum_{j=0}^{k} P_{j}\right)\right), \quad M_{0}:=\left\|\sum_{j=0}^{k} P_{j}\right\|_{\Omega}+\|g\|_{\Omega}, \quad N_{0}:=\operatorname{deg}\left(P_{k}\right)+1, \\
\varepsilon & :=\min \left\{\frac{\theta}{4} \inf _{z \in \partial \Omega} a\left(h(z)-\mathcal{R}^{p}\left(\sum_{j=0}^{k} P_{j}\right)(z)\right), \tau-\tau_{1}, \tau-\tau_{2}\right\}
\end{aligned}
$$

gives a polynomial $P_{k+1}$ such that $\operatorname{ord}\left(P_{k+1}\right)>\operatorname{deg}\left(P_{k}\right)$ and on $\partial \Omega$ the following inequalities hold

- $\frac{3}{4} \theta a\left(h-\mathcal{R}^{p}\left(\sum_{j=0}^{k} P_{j}\right)\right)<\mathcal{R}^{p}\left(P_{k+1}\right)<\frac{3}{4} a\left(h-\mathcal{R}^{p}\left(\sum_{j=0}^{k} P_{j}\right)\right)<a h$

- $\left|\mathcal{R}^{p}\left(\sum_{j=0}^{k+1} P_{j}\right)-\mathcal{R}^{p}\left(\sum_{j=0}^{k+1} P_{j}\right)-\mathcal{R}^{p}\left(P_{k+1}\right)\right|<\varepsilon$ 
- $\left|\mathcal{R}^{p}\left(g+\sum_{j=0}^{k+1} P_{j}\right)-\mathcal{R}^{p}\left(g+\sum_{j=0}^{k+1} P_{j}\right)-\mathcal{R}^{p}\left(P_{k+1}\right)\right|<\varepsilon$

- $\left|\mathcal{S}^{p}\left(P_{k+1}\right)\right|<\kappa\left(\frac{3}{4} a\left(h-\mathcal{R}^{p}\left(\sum_{j=0}^{k} P_{j}\right)\right)\right)^{\frac{1}{p}}<C\left(\mathcal{R}^{p}\left(P_{k+1}\right)\right)^{\frac{1}{p}}$.

Above inequalities imply that on $\partial \Omega$

$$
\begin{aligned}
& \mathcal{R}^{p}\left(\sum_{j=0}^{k+1} P_{j}\right)<\mathcal{R}^{p}\left(\sum_{j=0}^{k} P_{j}\right)+\mathcal{R}^{p}\left(P_{k+1}\right) \\
& \quad+\varepsilon<\mathcal{R}^{p}\left(\sum_{j=0}^{k} P_{j}\right)+a\left(h-\mathcal{R}^{p}\left(\sum_{j=0}^{k} P_{j}\right)\right)
\end{aligned}
$$

and

$$
\begin{aligned}
\mathcal{R}^{p}\left(\sum_{j=0}^{k+1} P_{j}\right)> & \mathcal{R}^{p}\left(\sum_{j=0}^{k} P_{j}\right)+\mathcal{R}^{p}\left(P_{k+1}\right)-\varepsilon \\
{[5 m m]>} & \mathcal{R}^{p}\left(\sum_{j=0}^{k} P_{j}\right)+\frac{3}{4} \theta a\left(h-\mathcal{R}^{p}\left(\sum_{j=0}^{k} P_{j}\right)\right) \\
& -\frac{\theta}{4} a\left(h-\mathcal{R}^{p}\left(\sum_{j=0}^{k} P_{j}\right)\right) \\
{[5 m m]=} & \mathcal{R}^{p}\left(\sum_{j=0}^{k} P_{j}\right)+\frac{1}{2} \theta a\left(h-\mathcal{R}^{p}\left(\sum_{j=0}^{k} P_{j}\right)\right) .
\end{aligned}
$$

By induction,

$$
\begin{aligned}
& \mathcal{R}^{p}\left(\sum_{j=0}^{k+1} P_{j}\right)<\mathcal{R}^{p}\left(\sum_{j=0}^{k} P_{j}\right)+\mathcal{R}^{p}\left(P_{k+1}\right) \\
& \quad+\varepsilon<\sum_{j=0}^{k+1} \mathcal{R}^{p}\left(P_{j}\right)+\tau_{1}+\varepsilon=\sum_{j=0}^{k+1} \mathcal{R}^{p}\left(P_{j}\right)+\tau \text { on } \partial \Omega
\end{aligned}
$$

and

$$
\begin{aligned}
\mathcal{R}^{p}\left(\sum_{j=0}^{k+1} P_{j}+g\right)< & \mathcal{R}^{p}\left(\sum_{j=0}^{k} P_{j}+g\right)+\mathcal{R}^{p}\left(P_{k+1}\right) \\
& +\varepsilon<\mathcal{R}^{p}(g)+\sum_{j=0}^{k+1} \mathcal{R}^{p}\left(P_{j}\right)+\tau_{2}+\varepsilon \\
& <\mathcal{R}^{p}(g)+\mathcal{R}^{p}\left(\sum_{j=0}^{k+1} P_{j}\right)+\tau \text { on } \partial \Omega .
\end{aligned}
$$

Similarly we get $\mathcal{R}^{p}\left(\sum_{j=0}^{k+1} P_{j}\right)>\sum_{j=0}^{k+1} \mathcal{R}^{p}\left(P_{j}\right)-\tau$ and $\mathcal{R}^{p}\left(\sum_{j=0}^{k+1} P_{j}+\right.$ $g)>\mathcal{R}^{p}(g)+\mathcal{R}^{p}\left(\sum_{j=0}^{k+1} P_{j}\right)-\tau$ on $\partial \Omega$. All the above implies that the polynomial $P_{k+1}$ is orthogonal to $P_{0}, P_{1}, \ldots, P_{k}$ and it satisfies conditions $(w 1)-(w 8)$.

Combining properties $(w 4)$ and $(w 5)$ with Dini's theorem one may conclude that $\mathcal{R}^{p}\left(\sum_{j=0}^{n} P_{j}\right)$ converges uniformly to $h$. Hence there exists $N \in \mathbb{N}$ such that 
$\mathcal{R}^{p}\left(\sum_{j=0}^{N} P_{j}\right)>\gamma h$ on $\partial \Omega$. Moreover, statement $(w 5)$ implies that

$$
h-\mathcal{R}^{p}\left(\sum_{j=0}^{k} P_{j}\right)<\left(1-\frac{1}{2} \theta a\right)\left(h-\mathcal{R}^{p}\left(\sum_{j=0}^{k-1} P_{j}\right)\right)<\left(1-\frac{1}{2} \theta a\right)^{k} h \text { on } \partial \Omega .
$$

Therefore, if $p \in(0,1]$, we have the following estimate on $\partial \Omega$

$$
\begin{aligned}
\left|\mathcal{S}^{p}\left(\sum_{j=0}^{N} P_{j}\right)\right| & \leq \sum_{j=0}^{N}\left|\mathcal{S}^{p}\left(P_{j}\right)\right| \stackrel{(w 9)}{<} C \sum_{j=0}^{N}\left(\mathcal{R}^{p}\left(P_{j}\right)\right)^{\frac{1}{p}} \\
& <C \sum_{j=0}^{N}\left(\frac{3}{4} a\left(h-\mathcal{R}^{p}\left(\sum_{j=0}^{k} P_{j}\right)\right)\right)^{\frac{1}{p}} \\
& <C \sum_{j=0}^{N}\left(\frac{3}{4} a\left(1-\frac{1}{2} \theta a\right)^{k} h\right)^{\frac{1}{p}} \\
& <C\left(\frac{3}{4} a h\right)^{\frac{1}{p}} \frac{1}{1-\left(1-\frac{1}{2} \theta a\right)^{\frac{1}{p}}}<C\left(\frac{3}{4} a h\right)^{\frac{1}{p}} \frac{2}{\theta a} \\
& <\frac{2 C}{\theta} h^{\frac{1}{p}} a^{\frac{1}{p}-1}
\end{aligned}
$$

and this finishes the proof.

Theorem 5.2 Let $\Phi$ be a strictly positive continuous function on $\partial \Omega$. There exists a holomorphic function $f=\sum_{n=0}^{\infty} p_{n}$, where $p_{n}$ are homogeneous polynomials, such that

1. $\mathcal{R}^{p}(f)=\Phi$ on $\partial \Omega$

2. every slice function of $\mathcal{S}^{p}(f)$ has a divergent series of Taylor coefficients with every exponent $s<\min \{1, p\}$, i.e. $\sum_{n=0}^{\infty}\left(\frac{\left|p_{n}(z)\right|}{\sqrt[p]{p n+1}}\right)^{s}=\infty, s<\min \{1, p\}, z \in \partial \Omega$

3. if $p \leq 1$, then $\mathcal{S}^{p}(f) \in \mathcal{C}(\bar{\Omega})$

4. if $p \in(1,2]$, then $\mathcal{S}^{p}(f) \in L^{2}(z \partial \mathbb{D}), z \in \partial \Omega$.

Proof Without loss of generality assume that $\sup _{z \in \partial \Omega}|\Phi(z)|<1$. Take constants $\theta \in$ $(0,1)$ and $C>0$ from Lemma 5.1. Inductively we will construct a sequence of orthogonal polynomials $\left\{Q_{k}\right\}_{k=0}^{\infty}$ with the following properties

(p1) $Q_{k}=\sum_{j=0}^{N_{k}} P_{k, j}$, where $P_{k, 0}, P_{k, 1}, \ldots, P_{k, N_{k}}$ are orthogonal polynomials

(p2) $\mathcal{R}^{p}\left(\sum_{m=0}^{k} Q_{m}\right)<\Phi$ on $\partial \Omega$

(p3) $\mathcal{R}^{p}\left(\sum_{m=0}^{k} Q_{m}\right)>\frac{1}{4}\left(\Phi-\mathcal{R}^{p}\left(\sum_{m=0}^{k-1} Q_{m}\right)\right)+\mathcal{R}^{p}\left(\sum_{m=0}^{k-1} Q_{m}\right)$ on $\partial \Omega$

(p4) $\mathcal{R}^{p}\left(P_{k, j}\right)<\left(\Phi-\mathcal{R}^{p}\left(\sum_{m=0}^{k-1} Q_{m}\right)\right)^{k}$ on $\partial \Omega, j=0,1, \ldots, N_{k}$ 
$(p 5)\left|\mathcal{S}^{p}\left(P_{k, j}\right)\right|<C\left(\mathcal{R}^{p}\left(P_{k, j}\right)\right)^{\frac{1}{p}}$ on $\partial \Omega, j=0,1, \ldots, N_{k}$

(p6) $\mathcal{R}^{p}\left(Q_{k}\right)-\left\|\Phi-\mathcal{R}^{p}\left(\sum_{m=0}^{k-1} Q_{m}\right)\right\|_{\partial \Omega}^{k}<\sum_{j=0}^{N_{k}} \mathcal{R}^{p}\left(P_{k, j}\right)<\mathcal{R}^{p}\left(Q_{k}\right)+$ $\left\|\Phi-\mathcal{R}^{p}\left(\sum_{m=0}^{k-1} Q_{m}\right)\right\|_{\partial \Omega}^{k}$ on $\partial \Omega$

(p7) $\left\|\mathcal{R}^{p}\left(Q_{k}\right)\right\|_{1, z \partial \mathbb{D}}>\frac{1}{2}\left\|\Phi-\mathcal{R}^{p}\left(\sum_{m=0}^{k-1} Q_{m}\right)\right\|_{1, z \partial \mathbb{D}}$

(p8) if $p \leq 1$, then $\left|\mathcal{S}^{p}\left(Q_{k}\right)\right|<\frac{2 C}{\theta}\left(\Phi-\mathcal{R}^{p}\left(\sum_{m=0}^{k-1} Q_{m}\right)\right)^{\frac{k}{p}-k+1}$ on $\partial \Omega$.

We begin by selecting $Q_{0} \equiv 0, N_{0}:=0, P_{0,0} \equiv 0$. For $k=1$ we apply Lemma 5.1 with

$$
a:=\inf _{z \in \partial \Omega} \Phi(z), \quad h:=\Phi, \quad \tau:=\inf _{z \in \partial \Omega} \Phi(z), \quad \gamma:=\frac{1}{2} .
$$

to obtain $N_{1} \in \mathbb{N}$ and orthogonal polynomials $P_{1,0}, P_{1,1}, \ldots, P_{1, N_{1}}$ such that the polynomial $Q_{1}:=\sum_{j=0}^{N_{1}} P_{1, j}$ has the following properties

- $\frac{1}{2} \Phi<\mathcal{R}^{p}\left(Q_{1}\right)<\Phi$ on $\partial \Omega$

- $\mathcal{R}^{p}\left(P_{1, j}\right)<\Phi$ on $\partial \Omega, j=0,1, \ldots, N_{1}$

- $\mathcal{R}^{p}\left(Q_{1}\right)-\|\Phi\|_{\partial \Omega}<\sum_{j=0}^{N_{1}} \mathcal{R}^{p}\left(P_{1, j}\right)<\mathcal{R}^{p}\left(Q_{1}\right)+\|\Phi\|_{\partial \Omega}$, on $\partial \Omega$

- $\left|\mathcal{S}^{p}\left(P_{1, j}\right)\right|<C\left(\mathcal{R}^{p}\left(P_{1, j}\right)\right)^{\frac{1}{p}}$ on $\partial \Omega, \quad j=0,1, \ldots, N_{1}$

- if $p \leq 1$, then $\left|\mathcal{S}^{p}\left(Q_{1}\right)\right|<\frac{2 C}{\theta}(\Phi)^{\frac{2}{p}-1} \leq \frac{2 C}{\theta} \Phi^{\frac{1}{p}}$ on $\partial \Omega$

- $\left\|\mathcal{R}^{p}\left(Q_{1}\right)\right\|_{1, z \partial \mathbb{D}}>\frac{1}{2}\|\Phi\|_{1, z \partial \mathbb{D}}$.

Observe that $Q_{1}$ satisfies conditions $(p 1)-(p 8)$. Then assume that the orthogonal polynomials $Q_{0}, Q_{1}, \ldots, Q_{k}$ with desired properties $(p 1)-(p 8)$ have been found. Lemma 5.1 applied to

$$
\begin{aligned}
M & :=\operatorname{deg}\left(Q_{k}\right)+1, \quad a:=\inf _{z \in \partial \Omega}\left(\Phi(z)-\mathcal{R}^{p}\left(\sum_{m=0}^{k} Q_{m}\right)(z)\right)^{k}, \\
h & :=\frac{3}{4}\left(\Phi-\mathcal{R}^{p}\left(\sum_{m=0}^{k} Q_{m}\right)\right), \\
\gamma & :=\frac{2}{3}, \quad \tau:=\frac{1}{8} \inf _{z \in \partial \Omega}\left(\Phi(z)-\mathcal{R}^{p}\left(\sum_{m=0}^{k} Q_{m}\right)(z)\right)^{k+1}
\end{aligned}
$$

produces $N_{k+1} \in \mathbb{N}$ and orthogonal polynomials $P_{k+1,0}, P_{k+1,1}, \ldots, P_{k+1, N_{k+1}}$ that are also orthogonal to the polynomials $Q_{0}, Q_{1}, \ldots, Q_{k}$. If we define $Q_{k+1}:=$ $\sum_{j=0}^{N_{k+1}} P_{k+1, j}$, then on $\partial \Omega$ it satisfies the following conditions 
a) $\operatorname{ord}\left(Q_{k+1}\right) \geq \operatorname{deg}\left(Q_{k}\right)+1$

b) $\mathcal{R}^{p}\left(P_{k+1, j}\right)<\left(\Phi-\mathcal{R}^{p}\left(\sum_{m=0}^{k} Q_{m}\right)\right)^{k+1}, j=0, \ldots, N_{k+1}$

c) $\left|\mathcal{S}^{p}\left(P_{k+1, j}\right)\right|<C\left(\mathcal{R}^{p}\left(P_{k+1, j}\right)\right)^{\frac{1}{p}}, \quad j=0, \ldots, N_{k+1}$

d) $\mathcal{R}^{p}\left(Q_{k+1}\right)-\left\|\Phi-\mathcal{R}^{p}\left(\sum_{m=0}^{k} Q_{m}\right)\right\|_{\partial \Omega}^{k+1}<\sum_{j=0}^{N_{k+1}} \mathcal{R}^{p}\left(P_{k+1, j}\right)<\mathcal{R}^{p}\left(Q_{k+1}\right)+$ $\left\|\Phi-\mathcal{R}^{p}\left(\sum_{m=0}^{k} Q_{m}\right)\right\|_{\partial \Omega}^{k+1}$

e) $\mathcal{R}^{p}\left(Q_{k+1}\right)<h=\frac{3}{4}\left(\Phi-\mathcal{R}^{p}\left(\sum_{m=0}^{k} Q_{m}\right)\right)$

f) $\mathcal{R}^{p}\left(Q_{k+1}\right)>\frac{1}{2}\left(\Phi-\mathcal{R}^{p}\left(\sum_{m=0}^{k} Q_{m}\right)\right)$

g) if $p \leq 1$, then $\left|\mathcal{S}^{p}\left(Q_{k+1}\right)\right|<\frac{2 C}{\theta}\left(\Phi-\mathcal{R}^{p}\left(\sum_{m=0}^{k} Q_{m}\right)\right)^{\frac{k+1}{p}-k}$

h)

$$
\begin{aligned}
\mathcal{R}^{p}\left(\sum_{m=0}^{k+1} Q_{m}\right) & <\mathcal{R}^{p}\left(\sum_{m=0}^{k} Q_{m}\right)+\mathcal{R}^{p}\left(Q_{k+1}\right)+2 \tau \\
& <\mathcal{R}^{p}\left(\sum_{m=0}^{k} Q_{m}\right)+\Phi-\mathcal{R}^{p}\left(\sum_{m=0}^{k} Q_{m}\right)=\Phi
\end{aligned}
$$

k)

$$
\begin{aligned}
\mathcal{R}^{p}\left(\sum_{m=0}^{k+1} Q_{m}\right) & >\mathcal{R}^{p}\left(\sum_{m=0}^{k} Q_{m}\right)+\mathcal{R}^{p}\left(Q_{k+1}\right)-2 \tau \\
& \geq \mathcal{R}^{p}\left(\sum_{m=0}^{k} Q_{m}\right)+\frac{1}{4}\left(\Phi-\mathcal{R}^{p}\left(\sum_{m=0}^{k} Q_{m}\right)\right) .
\end{aligned}
$$

Hence $Q_{k+1}$ has properties $(p 1)--(p 8)$.

Next observe that by $(p 2)$ and $(p 3), \lim _{n \rightarrow \infty} \mathcal{R}^{p}\left(\sum_{k=0}^{n} Q_{k}\right)=\Phi$ on $\partial \Omega$. In addition, property $(p 3)$ on $\partial \Omega$ implies the following estimate

$$
\begin{aligned}
\Phi-\mathcal{R}^{p}\left(\sum_{m=0}^{k} Q_{m}\right) & <\Phi-\frac{1}{4}\left(\Phi-\mathcal{R}^{p}\left(\sum_{m=0}^{k-1} Q_{m}\right)\right)-\mathcal{R}^{p}\left(\sum_{m=0}^{k-1} Q_{m}\right) \\
& =\frac{3}{4}\left(\Phi-\mathcal{R}^{p}\left(\sum_{m=0}^{k-1} Q_{m}\right)\right)<\left(\frac{3}{4}\right)^{k} \Phi
\end{aligned}
$$

Therefore it is clear that

$$
\mathcal{R}^{p}\left(Q_{k}\right)<\left(\frac{3}{4}\right)^{k}\|\Phi\|_{\partial \Omega} \text { on } \partial \Omega
$$


Let $q=\max \{1, p\}$. Observe that if $n \geq k$, then

$$
\begin{aligned}
\sup _{z \in \partial \Omega}\left(\mathcal{R}^{p}\left(\sum_{m=0}^{n} Q_{m}-\sum_{m=0}^{k} Q_{m}\right)(z)\right)^{\frac{1}{q}} & =\sup _{z \in \partial \Omega}\left(\mathcal{R}^{p}\left(\sum_{m=k+1}^{n} Q_{m}\right)(z)\right)^{\frac{1}{q}} \\
& \leq \sup _{z \in \partial \Omega}\left(\sum_{m=k+1}^{n}\left(\mathcal{R}^{p}\left(Q_{m}\right)(z)\right)^{\frac{1}{q}}\right) \\
& \stackrel{(15)}{<} \sum_{m=k+1}^{n}\left(\frac{3}{4}\right)^{\frac{m}{q}}\|\Phi\|_{\partial \Omega}^{\frac{1}{q}} \underset{n, k \rightarrow \infty}{\longrightarrow} 0,
\end{aligned}
$$

so $\left\{\sum_{m=0}^{n} Q_{m}\right\}_{n=0}^{\infty}$ is a Cauchy sequence in $\mathcal{H R}^{p}(\Omega)$ which, by Proposition 2.1, is convergent in $\mathcal{H} \mathcal{R}^{p}(\Omega)$. Hence we may define the function $f:=\sum_{k=0}^{\infty} Q_{k}=$ $\sum_{k=0}^{\infty} \sum_{j=0}^{N_{k}} P_{k, j}$. Proposition 2.1 implies that $f$ is holomorphic in $\Omega$. Moreover, $\mathcal{R}^{p}\left(\sum_{k=0}^{n} Q_{k}\right) \rightrightarrows \mathcal{R}^{p}(f)$ on $\partial \Omega$ as $n \rightarrow \infty$, so

$$
\mathcal{R}^{p}(f)(z)=\lim _{n \rightarrow \infty} \mathcal{R}^{p}\left(\sum_{k=0}^{n} Q_{k}\right)(z)=\Phi(z), \quad z \in \partial \Omega
$$

It follows from the property $(p 4)$ that for $z \in \partial \Omega$

$$
\left\|\Phi-\mathcal{R}^{p}\left(\sum_{m=0}^{k-1} Q_{m}\right)\right\|_{1, z \partial \mathbb{D}}^{-k}\left\|\mathcal{R}^{p}\left(P_{k, j}\right)\right\|_{1, z \partial \mathbb{D}}<1, \quad j=0, \ldots, N_{k} .
$$

Thus for $z \in \partial \Omega$ and $s<\min \{1, p\}$ we get that

$$
\begin{aligned}
\left\|\mathcal{R}^{p}\left(P_{k, j}\right)\right\|_{1, z \partial \mathbb{D}}^{s} & >\left\|\mathcal{R}^{p}\left(P_{k, j}\right)\right\|_{1, z \partial \mathbb{D}}^{s}\left(\left\|\Phi-\mathcal{R}^{p}\left(\sum_{m=0}^{k-1} Q_{m}\right)\right\|_{1, z \partial \mathbb{D}}^{-k}\left\|\mathcal{R}^{p}\left(P_{k, j}\right)\right\|_{1, z \partial \mathbb{D}}\right)^{p-s} \\
{[5 m m] } & =\left\|\Phi-\mathcal{R}^{p}\left(\sum_{m=0}^{k-1} Q_{m}\right)\right\|_{1, z \partial \mathbb{D}}^{-k(p-s)}\left\|\mathcal{R}^{p}\left(P_{k, j}\right)\right\|_{1, z \partial \mathbb{D}}^{p}, j=0, \ldots, N_{k} .
\end{aligned}
$$

Let $P_{k, j}(z)=\sum_{n \in I_{k, j}} p_{k, j, n}(z)$ be the homogeneous expansion of the polynomial $P_{k, j}$, where $I_{k, j}$ is the set of monomials' degrees of $P_{k, j}$. By construction, $I_{m, j} \cap I_{k, i}=$ 
$\emptyset$ for $(m, j) \neq(k, i)$. Moreover,

$$
\begin{aligned}
\left\|\mathcal{R}^{p}\left(P_{k, j}\right)\right\|_{1, z \partial \mathbb{D}}^{\frac{1}{q}} & =\left(\int_{\partial \mathbb{D}} \int_{0}^{1}\left|\sum_{n \in I_{k, j}} p_{k, j, n}(z \omega t)\right|^{p} d t d \lambda(\omega)\right)^{\frac{1}{q}} \\
& \leq \sum_{n \in I_{k, j}}\left(\int_{\partial \mathbb{D}} \int_{0}^{1}\left|p_{k, j, n}(z)\right|^{p} t^{p n} d t d \lambda(\omega)\right)^{\frac{1}{q}} \\
& =\sum_{n \in I_{k, j}} \frac{\left|p_{k, j, n}(z)\right|^{\frac{p}{q}}}{\sqrt[q]{p n+1}} .
\end{aligned}
$$

Observe that if $s<\min \{1, p\}$, then $\frac{s q}{p}<1$. Hence for $z \in \partial \Omega$ and $s<\min \{1, p\}$ we have the following estimate

$$
\begin{aligned}
& \sum_{k, j, n}\left(\frac{\left|p_{k, j, n}(z)\right|}{\sqrt[p]{p n+1}}\right)^{s}=\sum_{k, j, n}\left(\frac{\left|p_{k, j, n}(z)\right|^{\frac{p}{q}}}{\sqrt[q]{p n+1}}\right)^{\frac{s q}{p}} \\
& \geq \sum_{k, j}\left(\sum_{n \in I_{k, j}} \frac{\left|p_{k, j, n}(z)\right|^{\frac{p}{q}}}{\sqrt[q]{p n+1}}\right)^{\frac{s q}{p}} \geq \sum_{k, j}\left\|\mathcal{R}^{p}\left(P_{k, j}\right)\right\|_{1, z \partial \mathbb{D}}^{\frac{s}{p}} \\
& \stackrel{(16)}{>} \sum_{k=1}^{\infty}\left\|\Phi-\mathcal{R}^{p}\left(\sum_{m=0}^{k-1} Q_{m}\right)\right\|_{1, z \partial \mathbb{D}}^{-\frac{k(p-s)}{p}} \sum_{j=0}^{N_{k}}\left\|\mathcal{R}^{p}\left(P_{k, j}\right)\right\|_{1, z \partial \mathbb{D}} \\
& =\sum_{k=1}^{\infty}\left\|\Phi-\mathcal{R}^{p}\left(\sum_{m=0}^{k-1} Q_{m}\right)\right\|_{1, z \partial \mathbb{D}}^{-\frac{k(p-s)}{p}} \sum_{j=0}^{N_{k}} \int_{\partial \mathbb{D}} \mathcal{R}^{p}\left(P_{k, j}\right)(\omega z) d \lambda(\omega) \\
& \stackrel{(p 6)}{>} \sum_{k=1}^{\infty}\left\|\Phi-\mathcal{R}^{p}\left(\sum_{m=0}^{k-1} Q_{m}\right)\right\|_{1, z \partial \mathbb{D}}^{-\frac{k(p-s)}{p}}\left(\int_{\partial \mathbb{D}} \mathcal{R}^{p}\left(Q_{k}\right)(\omega z) d \lambda(\omega)\right. \\
& \left.-\left\|\Phi-\mathcal{R}^{p}\left(\sum_{m=0}^{k-1} Q_{m}\right)\right\|_{\partial \Omega}^{k}\right) \\
& \geq \sum_{k=1}^{\infty}\left\|\Phi-\mathcal{R}^{p}\left(\sum_{m=0}^{k-1} Q_{m}\right)\right\|_{1, z \partial \mathbb{D}}^{-\frac{k(p-s)}{p}}\left\|\mathcal{R}^{p}\left(Q_{k}\right)\right\|_{1, z \partial \mathbb{D}}-\sum_{k=1}^{\infty}\left(\frac{3}{4}\right)^{\frac{(k-1) k s}{p}} \\
& \stackrel{(p 7)}{>} \sum_{k=1}^{\infty} \frac{1}{2}\left\|\Phi-\mathcal{R}^{p}\left(\sum_{m=0}^{k-1} Q_{m}\right)\right\|_{1, z \partial \mathbb{D}}^{-\frac{k(p-s)}{p}+1}-\sum_{k=1}^{\infty}\left(\frac{3}{4}\right)^{\frac{(k-1) k s}{p}} \\
& \geq \sum_{k>\frac{p}{p-s}} \frac{1}{2}-\sum_{k=1}^{\infty}\left(\frac{3}{4}\right)^{\frac{(k-1) k s}{p}}=\infty \text {. }
\end{aligned}
$$

Since the last series diverges, so does the series $\sum_{k, j, n}\left(\frac{\left|p_{k, j, n}(z)\right|}{\sqrt[p]{p n+1}}\right)^{s}$. 
If $p \leq 1$, then property ( $p 8$ ) gives the estimate in $\bar{\Omega}$

$$
\begin{aligned}
\sum_{k=1}^{\infty}\left|\mathcal{S}^{p}\left(Q_{k}\right)\right| & \leq \frac{2 C}{\theta} \sum_{k=1}^{\infty}\left\|\Phi-\mathcal{R}^{p}\left(\sum_{m=0}^{k-1} Q_{m}\right)\right\|_{\partial \Omega}^{\frac{k}{p}-k+1} \\
& \leq \frac{2 C}{\theta} \sum_{k=1}^{\infty}\left(\frac{3}{4}\right)^{k-1}\|\Phi\|_{\partial \Omega}<\infty
\end{aligned}
$$

which implies that $\mathcal{S}^{p}(f) \in \mathcal{C}(\bar{\Omega})$.

Since polynomials $P_{k, 0}, P_{k, 1}, \ldots, P_{k, N_{k}}$ are orthogonal, so are $\mathcal{S}^{p}\left(P_{k, 0}\right)$, $\mathcal{S}^{p}\left(P_{k, 1}\right), \ldots, \mathcal{S}^{p}\left(P_{k, N_{k}}\right)$. Then for $p \in(1,2]$ consider the integral

$$
\begin{aligned}
& \int_{\partial \mathbb{D}}\left|\mathcal{S}^{p}(f)(\omega z)\right|^{2} d \lambda(\omega)=\int_{\partial \mathbb{D}}\left|\sum_{k=0}^{\infty} \sum_{j=0}^{N_{k}} \mathcal{S}^{p}\left(P_{k, j}\right)(\omega z)\right|^{2} d \lambda(\omega) \\
& =\sum_{k=0}^{\infty} \sum_{j=0}^{N_{k}} \int_{\partial \mathbb{D}}\left|\mathcal{S}^{p}\left(P_{k, j}\right)(\omega z)\right|^{2} d \lambda(\omega) \\
& \stackrel{(p 5)}{\leq} C^{2} \sum_{k=0}^{\infty} \sum_{j=0}^{N_{k}} \int_{\partial \mathbb{D}}\left(\mathcal{R}^{p}\left(P_{k, j}\right)(\omega z)\right)^{\frac{2}{p}} d \lambda(\omega) \\
& \leq C^{2} \sum_{k=0}^{\infty} \sum_{j=0}^{N_{k}} \int_{\partial \mathbb{D}} \mathcal{R}^{p}\left(P_{k, j}\right)(\omega z) d \lambda(\omega) \\
& \stackrel{(p 6)}{\leq} C^{2} \sum_{k=1}^{\infty}\left(\int_{\partial \mathbb{D}} \mathcal{R}^{p}\left(Q_{k}\right)(\omega z) d \lambda(\omega)\right. \\
& \left.+\left\|\Phi-\mathcal{R}^{p}\left(\sum_{m=0}^{k-1} Q_{m}\right)\right\|_{\partial \Omega}^{k}\right) \\
& \stackrel{(15)}{<} C^{2} \sum_{k=1}^{\infty}\left(\left(\frac{3}{4}\right)^{k}\|\Phi\|_{\partial \Omega}+\left(\frac{3}{4}\right)^{(k-1) k}\right)<\infty \text {. }
\end{aligned}
$$

Hence $\mathcal{S}^{p}(f) \in L_{2}(z \partial \mathbb{D})$ for $p \in(1,2], z \in \partial \Omega$. The proof is now complete.

Observe that for $p=2$ due to orthogonality of $p_{k, j, n}$ in (17) each slice function of $\mathcal{S}^{2}(f)$ additionally has divergent series of Taylor coefficients with every exponent $s<2$.

Data Availibility Data sharing not applicable to this article as no datasets were generated or analysed during the current study.

Open Access This article is licensed under a Creative Commons Attribution 4.0 International License, which permits use, sharing, adaptation, distribution and reproduction in any medium or format, as long as you give appropriate credit to the original author(s) and the source, provide a link to the Creative Commons licence, 
and indicate if changes were made. The images or other third party material in this article are included in the article's Creative Commons licence, unless indicated otherwise in a credit line to the material. If material is not included in the article's Creative Commons licence and your intended use is not permitted by statutory regulation or exceeds the permitted use, you will need to obtain permission directly from the copyright holder. To view a copy of this licence, visit http://creativecommons.org/licenses/by/4.0/.

\section{References}

1. Aleksandrov, A.B.: The existence of inner functions in the ball. Mat. Sb. (N.S.) 46(2), 147-163 (1982)

2. Karaś, M., Kot, P.: Divergent series of Taylor coefficients on almost all slices. Bull. Belg. Math. Soc. Simon Stevin 26, 1-9 (2019)

3. Kot, P.: On analytic functions with divergent series of Taylor coefficients. Complex Anal. Oper. Theory 12(5), 1237-1249 (2017)

4. Kot, P.: A holomorphic function with given almost all boundary values on a domain with holomorphic support function. J. Convex Anal. 14(4), 693-704 (2007)

5. Kot, P.: Homogeneous polynomials on strictly convex domains. Proc. Amer. Math. Soc. 135, 38953903 (2007)

6. Kot, P.: Bounded holomorphic functions with given maximal modulus on all circles. Proc. Am. Math. Soc. 137, 179-187 (2009)

7. Kot, P.: Radon inversion problem for holomorphic functions on strictly pseudoconvex domains. Bull. Belg. Math. Soc. Simon Stevin 17, 623-640 (2010)

8. Kot, P.: About boundary values in $A(\Omega)$. Trans. Am. Math. Soc. 363(8), 4063-4079 (2011)

9. Løw, E.: A construction of inner functions on the unit ball in $\mathbb{C}^{p}$. Inventiones mathematicae 67, 223-229 (1982)

10. Rudin, W.: Function theory in the unit ball of $\mathbb{C}^{n}$, Reprint of the 1980 edition. In: Classics in Mathematics. Springer, Berlin (2008)

11. Ryll, J., Wojtaszczyk, P.: On homogeneous polynomials on a complex ball. Trans. Amer. Math. Soc. 276, 107-116 (1983)

12. Wojtaszczyk, P.: On functions in the ball algebra. Proc. Am. Math. Soc. 85(2), 184-186 (1982)

Publisher's Note Springer Nature remains neutral with regard to jurisdictional claims in published maps and institutional affiliations. 